\title{
Multicriteria Based Next Forwarder Selection for Data Dissemination in Vehicular Ad Hoc Networks Using Analytical Network Process
}

\author{
Shahid Latif, ${ }^{1}$ Saeed Mahfooz, ${ }^{2}$ Bilal Jan, ${ }^{1}$ Naveed Ahmad, ${ }^{2}$ \\ Haleem Farman, ${ }^{2}$ Murad Khan, ${ }^{1}$ and Huma Javed ${ }^{2}$ \\ ${ }^{1}$ Department of Computer Science \& IT, Sarhad University of Science and IT, Peshawar, Pakistan \\ ${ }^{2}$ Department of Computer Science, University of Peshawar, Peshawar, Pakistan \\ Correspondence should be addressed to Bilal Jan; bilal.csit@suit.edu.pk
}

Received 7 April 2017; Revised 24 July 2017; Accepted 13 August 2017; Published 1 October 2017

Academic Editor: Juan C. Leyva

Copyright (C) 2017 Shahid Latif et al. This is an open access article distributed under the Creative Commons Attribution License, which permits unrestricted use, distribution, and reproduction in any medium, provided the original work is properly cited.

Vehicular ad hoc network (VANET) is a wireless emerging technology that aims to provide safety and communication services to drivers and passengers. In VANETs, vehicles communicate with other vehicles directly or through road side units (RSU) for sharing traffic information. The data dissemination in VANETs is a challenging issue as the vehicles have to share safety critical information in real time. The data distribution is usually done using broadcast method resulting in inefficient use of network resources. Therefore, to avoid the broadcast storm and efficiently use network resources, next forwarder vehicle (NFV) is selected to forward data to nearby vehicles. The NFV selection is based on certain parameters like direction, distance, and position of vehicles, which makes it a multicriteria decision problem. In this paper, analytical network process (ANP) is used as a multicriteria decision tool to select the optimal vehicle as NFV. The stability of alternatives (candidate vehicles for NFV selection) ranking is checked using sensitivity analysis for different scenarios. Mathematical formulation shows that ANP method is applicable for NFV selection in VANETs. Simulation results show that the proposed scheme outperforms other state-of-the-art data dissemination schemes in terms of reachability, latency, collisions, and number of transmitted and duplicate data packets.

\section{Introduction}

Vehicular ad hoc network (VANET) is a wireless technology that uses moving vehicles as nodes to create a network for information exchange. These networks are decentralized where every vehicle periodically broadcasts its information, that is, direction, position, speed, and so on, through periodic beaconing to deliver a message to the neighbor vehicles inside a concern area [1]. Most importantly, critical situations such as accidents or traffic jams make use of a vehicle's information to disseminate warning messages to all other distant vehicles of network. Communication in VANETs is usually between vehicles, where they directly transfer information to other vehicle and referred as vehicle-to-vehicle (V2V) communication. Moreover, vehicles might communicate through road side unit (usually installed at light pole or traffic light) with other vehicles, known as vehicle-to-infrastructure (V2I) communication. [2]. The VANETs comprise a large set of applications that can be categorized mainly in two groups: safety and nonsafety applications. Safety applications include situation awareness, traffic services, and cooperative warning messages. On the other hand, nonsafety applications include infotainment, traffic information, road monitoring, automated roadways, environmental protection, and many more [3]. These applications assist the academia and industry to develop and implement new products and procedures.

The data dissemination in VANETs is a broadcastoriented message distribution process, where information is required to be sent to group of vehicles instead of individual vehicle. The broadcast-oriented data dissemination leads to several critical problems such as broadcast storm, network partitions, and inefficient use of energy resources. The broadcast storm in VANETs arises during high traffic densities where every vehicle has the authority to retransmit the data packet inside a network simultaneously as shown in Figure 1. Therefore, such Flooding-based data 


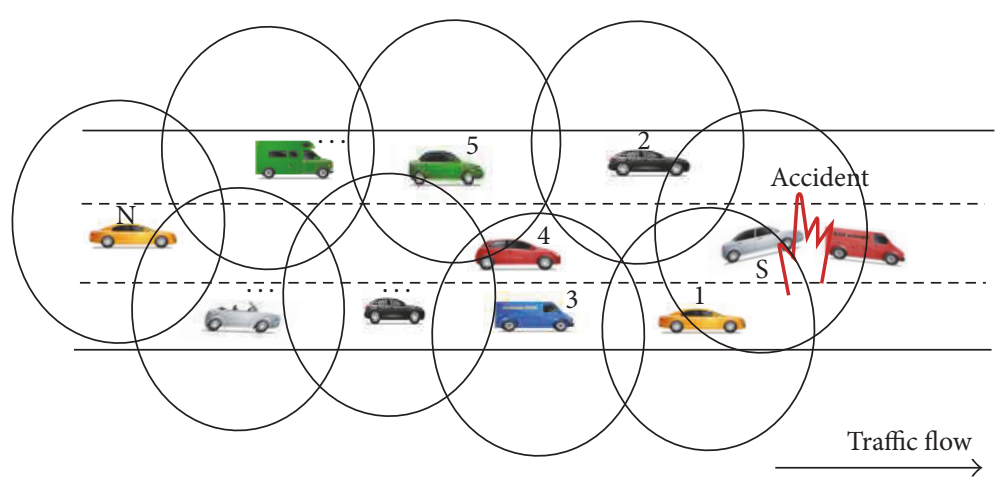

FIGURE 1: Broadcast storm problem in VANETs.

dissemination techniques lead to redundant transmissions, contention, delivery delay, high data packet loss ratio, and immense traffic load. To cope up the problem of broadcast storm, an optimal next forwarder vehicle selection process is required that should ensure effective transmission between vehicles by eliminating the broadcast. In literature, many data dissemination techniques are proposed to ensure effective and efficient transmission to reduce the messages exchanged and avoid redundant messages.

In this paper, a novel data dissemination protocol for highway scenario in VANETs is proposed. The forwarding strategy (selection of NFV) is based on direction, position, and distance of vehicles with respect to source vehicle. Moreover, on the basis of these parameters it assigns the forwarding priorities to all vehicles inside transmission region. Highest forwarding priority will be assigned to the farthest vehicle(s) inside ideal segments moving in same direction to the source. The vehicle with shortest waiting time will retransmit data packet to carry on the data dissemination process. It avoids the retransmission of already scheduled data packets in order to avoid the broadcast storm. This protocol also employs store-carry-forward mechanisms to deal with network partitions. In this paper, the next forward vehicle is selected among candidate vehicles using a multicriteria decision tool known as analytical network process. The ANP method is used as it deals with the interdependencies among elements and deals with feedback as well, which was not offered in previous version named analytical hierarchy process (AHP). This paper also presents a mathematical model to compute the priorities of vehicles inside a network. Finally, a case study consists of a highway VANETs scenario used to implement the ANP model.

The rest of the paper is organized as follows. Section 2 illustrates related work in detail about VANET in general and data dissemination protocols in particular. The ANP method for NFV selection problem is discussed in Section 3. In Section 4, the criteria and their interrelation used for compiling selection decision are discussed. A multicriteria decision framework based on ANP model is presented in Section 5. Simulation results carried out in VEINS for different parameters are discussed in Section 6. Finally, Section 7 concludes the paper by discussing limitations and future directions.

\section{Background Knowledge and Related Work}

A survey conducted in 2010, according to the report number of automobiles operating on roads, all over the world has reached to remarkable count of one billion from 500 million in just 24 years since 1986 [4]. The survey in 2014 concluded the mark of 1.2 billion entities on roads and we believe it will reach to 2 billion by 2035 [5]. Such huge traffic brought about 1.25 million fatalities per year and, without precautions or systems, by 2030 road traffic fatalities will become the 7 th leading cause of deaths [6]. Based on these statistics the academia, research, and industrial community introduce an intelligent and ultimate solution. Thus, Intelligent Transportation Systems (ITS) are deployed to enhance safety, improve mobility, and reduce congestion. These systems integrate wide range of wired and wireless communication, computing, and modern electronics technologies. Among these technologies, vehicular communication has extensively grabbed the attention of research, academia, and industrial community [7].

VANETs, an important unit of ITS, are the decentralized, infrastructure-less and self-organized networks of vehicles. It allows vehicles equipped with communication systems, onboard processing devices, sensors, and navigation capabilities to establish a temporary wireless network on roads without any fixed infrastructure. In VANETs, every vehicle is a mobile node that collects and forwards the important traffic information to other nodes of network. VANETs use diverse communication model such as unicast, multicast, geocast, and broadcast for reliable and uninterrupted communication. Broadcast-oriented communication is more suitable for vehicular environment which requires information distribution to every vehicle of the network. In fact, broadcasting is a data dissemination process in which a vehicle has to broadcast the information to its neighbor vehicles.

The literature presents an extensive and prosperous work on data dissemination process and corresponding protocols. Generally, these protocols address the two key problems, that is, the broadcast storm [8-12] and the network partitions [13, 14]. Flooding is the simplest way to broadcast the information inside the concern area (CA) of a network. It is very simple to implement and provides best performance in sparse traffic conditions as it delivers the data packet very quickly to the 
vehicles of a network. Major drawback of this technique is the simultaneous retransmissions of same data packet by all vehicles of a network. Consequently, the network may find broadcast storm. Also growing number of redundant transmissions begin augmented load and consequently wastage of limited bandwidth.

2.1. Data Dissemination Protocols. The data transmission plays important part in minimizing number of messages, avoiding redundant messages, and reducing delay in VANETs. Therefore, numbers of data dissemination protocols are proposed in literature $[13,15]$. The forwarding mechanism is proposed in various formats based on location, position, distance, network topology, delay, density, and so on discussed in [16]. In this section of the paper, various well-known data dissemination protocols along with their forwarding strategies are discussed. Adaptive Information Dissemination (AID) [13] protocol uses a counter-based approach to decide the next forwarder vehicle. In AID, every vehicle counts how many times it has received the same data packet in specified time period. It minimizes the broadcast storm as several vehicles decide to discard the received data packet.

Broadcast Communication (BROADCOMM) [17] protocol forwards the data packets (emergency warning information) on the basis of geographical routing. In BROADCOMM, the highway is divided into virtual movable cells that move with vehicle's movement. It uses two-level hierarchical structure to select the NFV termed as Cell Reflector (CR). At first level, vehicles of a cell communicate with each other and with neighbor cell vehicles, while at second level, a vehicle closer to geographical center of its own cell is selected as the $\mathrm{CR}$ responsible for onward transmissions and communications.

Distance Based Relay Selection (DBRS) [14] protocol uses distance-based approach to decide next forwarder vehicle. It uses a relay selection algorithm for selection of NFV. In DBRS, every vehicle is assigned a waiting time according to the distance between transmitting vehicle and itself. Farthest vehicle will find the shortest waiting time and will be responsible for data packet retransmission. Every other vehicle which has scheduled the data packet for further retransmission will cancel it in order to avoid the broadcast storm. DBRS may produce high data delivery delays whenever no border vehicle will be discovered. DRIFT [11] protocol forwarding strategy is based on both the position and distance information of vehicles. It divides the transmission range of a vehicle in different zones. Vehicles located inside the preference zone are believed to be the best options as $\mathrm{NFV}$ (s) to eliminate the broadcast storm. DRIFT also addresses and tackles the network partitions using store-carry-forward mechanism.

Irresponsible Forwarding (IF) [18] protocol is distributed protocol based on distance and density of nearby vehicles. The vehicles calculate their distance from the source vehicle and density of all neighbor vehicles to settle their forwarding probabilities. Moreover, vehicles compare their forwarding probabilities and avoid data packet transmission if they have low probabilities compared to other vehicles. The authors claim that their proposed protocol controls the transmission rate and consequently mitigates the broadcast storm.

Distributed Vehicular broadCAST (DV-CAST) [8] protocol uses local topology for selection of NFV during data dissemination process in VANETs. DV-CAST forwarding strategy depends on one-hop neighbor information collected though periodic beaconing. It performs the dissemination process in three steps: (1) detecting all neighbor vehicles, gathering relevant information though periodic beaconing, and creating local topology, (2) using weighted p-persistence technique to handle the broadcast storm during high traffic densities, and (3) using store-carry-forward technique to deal with network partitions during low traffic densities. Similarly, Simple and Robust Dissemination (SRD) [19] protocol is an improvement over DV-CAST. In SRD, a data packet is transmitted in recommended direction of interest (DoI).

MobiCast [9] and MobiCast-Carry-Forward [10] protocols are designed for some specific applications of VANETs that involve spatiotemporal multicast phenomenon. Their forwarding strategies are based on position and timer based approaches. MobiCast-Carry-Forward protocol makes use of carry-\&-forward mechanism that turns out in larger delays but it improves the performance of actual MobiCast protocol in terms of successful dissemination rate and network overhead. A major weakness of these protocols is the selection of zone of relevance $(\mathrm{ZoR})$, zone of forwarding $(\mathrm{ZoF})$, and vehicles inside the ZoF using a complex process. Weighted p-persistence, Slotted 1-persistence and Slotted p-persistence [12] are the probabilistic information dissemination protocols that use position-based approaches for NFV selection. In these protocols, vehicles calculate their forwarding probability before retransmitting the received data packet using information collected through global positioning systems.

2.2. A Qualitative Comparison of Reviewed Protocols. The qualitative comparison among the aforementioned data dissemination protocols for VANETs is described in Table 1. The comparison is based on three types of criteria: forwarding strategy, objective, and scenario. Forwarding strategy presents the method(s) used by protocols to route the data packet towards the target. As stated previously, the forwarding strategy of data dissemination protocols may depend on different types of information including location, position, distance, network topology, delay, and density. Most of the protocols discussed in this paper use position or distance information of vehicles to determine how to route data packet. It is also observed that some protocols use network topology, delay, and density or direction information to make decisions about data forwarding. Some protocols make use of carry-and-forward (buffering) approach to deal with network partitions. Objective criterion defines the main objectives addressed by the protocol based on some serious issues and challenges. The scenario criterion represents the operating environments of each protocol.

2.3. Multicriteria Decision Analysis. The multicriteria decision analysis (MCDA) techniques are used to assist the people in making decisions by providing stepping-stones for 


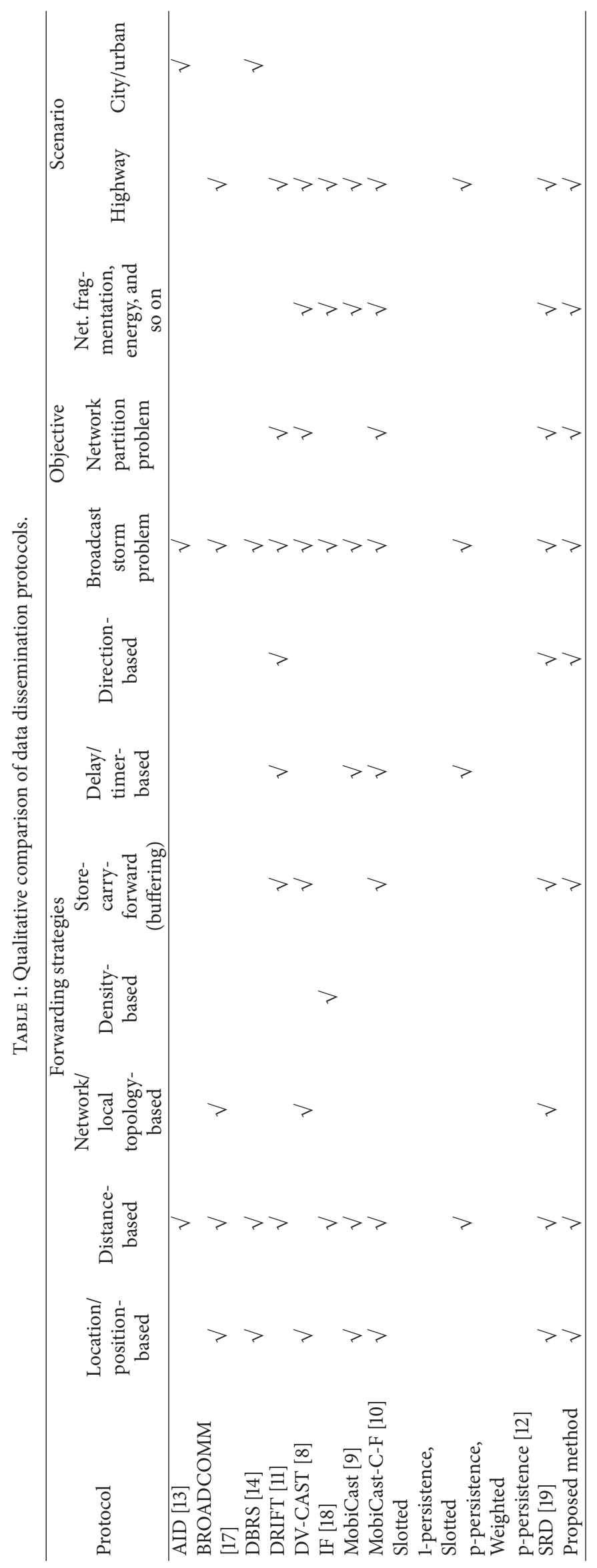


finding compromised outcomes from their decision process. These techniques require some subjective information from decision makers to find the solution for their decisions. The MCDA is equally important in every field of knowledge where someone needs to make a significant decision, for example, mathematics, engineering, informatics, economics, and management. In literature, different techniques are developed to provide solutions to multicriteria decision problems [20, 21]. Decision problems can be categorized in several classes such as choice-based, sorting-based, rankingbased, description-based, design-based, and eliminationbased problems [22-24]. To solve these decision problems many techniques have been developed like AHP, ANP, TOPSIS, ELECTRE, DEA, MAUT/UTA, PROGETHEE, and so on. Similarly to structure and solve these problems many software programs are designed and proposed including Decision Lab, D-Sight, DECERNS, Right Choice, MakeItRational, ExpertChoice, Super Decision, M-MACBETH, DEASolver, and Smart Picker Pro [25].

\section{Analytical Network Process}

The analytical network process was introduced by Saaty [26]; it was developed on the basis of AHP [27] as already discussed in Section 2. AHP is a rank or priority measurement model that needs subjective information (preferences or scores) as inputs on multiple criteria to evaluate all suggested alternatives for achieving the stated goal. In addition, if criteria of decision are possibly dependent on its alternatives (mandatory dependence of criteria and alternatives) then we are having a decision with dependence and feedback. To deal with such decisions and to determine best track of action analytical network process (ANP) [26] was developed. ANP structures complex multicriteria decision problems as a network of different clusters with dependencies between elements within a cluster or between clusters and provides feedback as well. Every cluster is a group of various elements connected to each other. The ANP model is used in different fields for decision making such as in the field of social sciences, mathematical, commerce, and economical and physical science [28-30]. The generalized steps of ANP method are summarized as follows:

(1) The problem is identified and divided into to subproblem by mentioning the objective(s), criteria/subcriteria, and possible alternatives in an understandable way. Objective is basically the goal that we want to achieve, criteria are the parameters that are involved in decision making, and alternatives are the possible options for selection.

(2) Develop the hierarchy and corresponding general network (model) of clusters along with their elements in order to obtain the priorities of criteria through pair wise comparison matrices shown in Figure 2. Connect the clusters and their elements according to their inner and outer dependencies with respect to direction of influence (use arrows drawn from one to another cluster).

(3) Determine a fundamental qualitative scale of influence (importance) to be followed in the analysis of clusters and their elements. A qualitative scale for comparing two clusters or their elements in ANP method is presented by [31] as shown in Table 2. This qualitative scale is converted to a quantitative scale ranging from 1 to 9 .

(4) Once the scaling is done, elements of different clusters are pairwise compared. A matrix is created by comparing $i$ th row with $j$ th column. According to the quantitative scale, 1 represents equal importance while 9 shows most importance.

(5) Construct supermatrix for each criterion by placing all elements of each cluster on the left as well as at the top of that super matrix. Also put the priorities obtained from pairwise comparisons in appropriate positions in super matrix. In general, supermatrix of a network can be shown as in

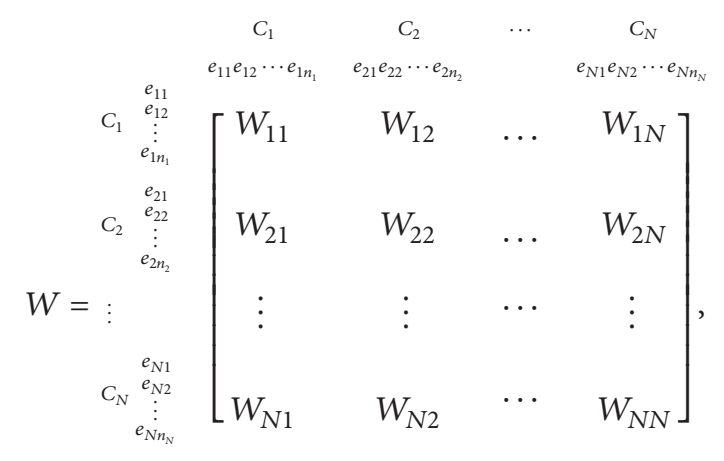

where $W_{i j}=1 / W_{j i} \forall i, j$.

(6) Calculate the relative priorities of criteria through the principal Eigen value and corresponding principal Eigenvector of a matrix. Every element of the matrix is normalized using the concepts of geometric mean (2) and termed as weights of the criteria or subcriteria. This will result in a weighted stochastic supermatrix.

$$
\left(\prod_{i=1}^{n} W i\right)=\sqrt[n]{w 1 * w 2 * w 3 \cdots w n}
$$

(7) Compute the consistency of the weighted supermatrix after pairwise comparison. The consistency index (CI) of a matrix can be calculated using

$$
\mathrm{CI}=\frac{\left(\lambda_{\max }-n\right)}{(n-1)},
$$

where $n$ is the order of matrix and $\lambda_{\max }$ is the Eigen value of that matrix. It can be calculated using

$$
A_{w}=\lambda_{\max } w
$$

where " $w$ " is the Eigenvector of matrix " $A$." Similarly, the consistency ratio (CR) of a matrix can be calculated using

$$
\mathrm{CR}=\frac{\mathrm{CI}}{\mathrm{RI}}
$$


TABLE 2: Fundamental scale.

\begin{tabular}{|c|c|}
\hline Intensity/level & Description \\
\hline 1 & $\begin{array}{l}\text { Equal Importance (assign equal importance to } \\
\text { both actions, i.e., both contribute equally to } \\
\text { objectives) }\end{array}$ \\
\hline 2 & Intermediate values (weak or slightly importance) \\
\hline 3 & $\begin{array}{l}\text { Moderate importance (slightly support one } \\
\text { action over another) }\end{array}$ \\
\hline 4 & Intermediate values (moderate plus importance) \\
\hline 5 & $\begin{array}{l}\text { Strong importance (strongly support one action } \\
\text { over another) }\end{array}$ \\
\hline 6 & Intermediate values (strong plus importance) \\
\hline 7 & $\begin{array}{l}\text { Very strong importance (An action is supported } \\
\text { very strongly over another) }\end{array}$ \\
\hline 8 & Intermediate values (very strong importance) \\
\hline 9 & $\begin{array}{l}\text { Extreme importance (support one action over } \\
\text { another with the highest confirmation level) }\end{array}$ \\
\hline Reciprocal & $\begin{array}{l}\text { Use reciprocal for inverse comparison; that is, if } \\
\text { an action "A" is extremely more important than } \\
\text { another action "B" (rated 9), then "B" is } \\
\text { extremely less important than "A" (rated 1/9) and } \\
\text { so on }\end{array}$ \\
\hline
\end{tabular}

Random consistency Index (RI) is an average random index computed by Saaty for $n \leq 10$ as shown in Table 3. The RI values for consecutive number above $10(n>10)$ demonstrate very little difference. Hence these numbers are not important to be mentioned over here. Moreover, the consistency ratio (CR) is needed to be less than or equal to 0.10 which is an acceptable level of inconsistency [32].

(8) Calculate the limit priorities and put them in supermatrix in idealized form, (while the priority vectors should be put in normalized form).

(9) Obtain the complete supermatrix by combining the entire work in a single matrix. The supermatrix may be either unweighted or weighted supermatrix. In case the column sum of supermatrix is greater than 1 then it is unweighted supermatrix. Normalize it until its column sum turn out to be less than or equal to 1 (weighted supermatrix).

(10) Convert the weighted supermatrix into the limit matrix.

(11) Determine the appropriate alternative from limit matrix. The generic form of limit matrix is shown in Table 4 .

(12) Finally, the sensitivity analysis is carried out to check the stability of alternatives ranking. This is not mandatory but highly recommended.

\section{Criteria for Selection Decision and Their Interrelation}

Qualitative comparison of data dissemination protocols as presented in Table 1 indicates that most of the protocols use
TABLE 3: Random index.

\begin{tabular}{lcccccccccc}
\hline Order & 1 & 2 & 3 & 4 & 5 & 6 & 7 & 8 & 9 & 10 \\
\hline R.I. & 0 & 0 & 0.52 & 0.89 & 1.11 & 1.25 & 1.35 & 1.40 & 1.45 & 1.49 \\
\hline
\end{tabular}

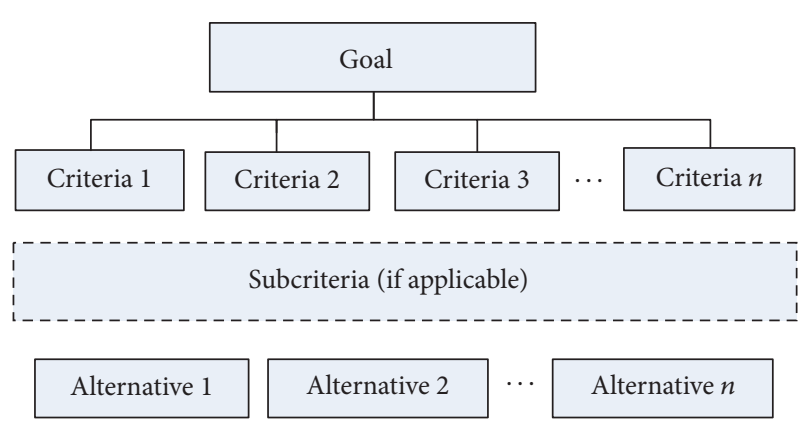

FIGURE 2: Network structure.

either position or distance-based forwarding strategy. The position-based protocols use position information of vehicles to determine how to route data, while distance-based protocols use the farthest vehicle (border vehicle) in the planned direction to route data. Therefore, every farthest/border vehicle on all sides of the source/transmitting vehicle is equally important to be selected as the NFV. In this paper, we present a novel data dissemination protocol for vehicle-tovehicle $(\mathrm{V} 2 \mathrm{~V})$ communication in VANETs. The proposed method classifies all vehicles inside the transmission range in three groups and assigns planned priorities. One vehicle selected as the NFV from highest priority group is enough to carry on the data dissemination process. The proposed method uses local information of neighbor vehicle obtained via global position system (GPS) and a navigation system. It mainly uses three types of information (summarized in Table 5) regarding neighbor vehicles to make a decision of NFV selection for data dissemination in VANETs. The parameters used are as follows:

(i) Direction. The proposed method assigns the lowest forwarding priority to the vehicles moving in opposite direction to a source or transmitting vehicle. The opposite direction vehicles become significant for data dissemination process only during low traffic densities. Moreover, vehicles moving away from the source/transmitting vehicle are also considered less significant than the vehicles moving towards a source/transmitting vehicle and therefore assigned with smaller forwarding probabilities.

(ii) Position. The proposed method categorizes vehicles inside the transmission range in three groups. It assigns highest forwarding probability to the vehicles positioned in ideal segment, that is, right behind the source/transmitting vehicle shown in Figure 3 . The vehicles positioned in normal segment are assigned with low priorities while vehicles positioned inside the ahead segment are assigned with lowest forwarding probabilities. 
TABLE 4: Limit matrix.

\begin{tabular}{|c|c|c|c|c|c|c|c|c|c|}
\hline \multirow{2}{*}{ Cluster node labels } & \multicolumn{4}{|c|}{ Criteria } & \multicolumn{4}{|c|}{ Alternatives } & \multirow{2}{*}{$\begin{array}{c}\text { Goal } \\
\text { Best option }\end{array}$} \\
\hline & $\mathrm{C} 1$ & $\mathrm{C} 2$ & $\mathrm{C} 3$ & $\mathrm{C} 4$ & $\mathrm{~A} 1$ & $\mathrm{~A} 2$ & $\mathrm{~A} 3$ & $\mathrm{~A} 4$ & \\
\hline \multicolumn{10}{|l|}{ Criteria } \\
\hline \multicolumn{10}{|l|}{$\mathrm{C} 1$} \\
\hline \multicolumn{10}{|l|}{$\mathrm{C} 2$} \\
\hline \multicolumn{10}{|l|}{$\mathrm{C} 3$} \\
\hline \multicolumn{10}{|l|}{$\mathrm{C} 4$} \\
\hline \multicolumn{10}{|l|}{ Alternatives } \\
\hline \multicolumn{10}{|l|}{$\mathrm{A} 1$} \\
\hline \multicolumn{10}{|l|}{$\mathrm{A} 2$} \\
\hline \multicolumn{10}{|l|}{$\mathrm{A} 3$} \\
\hline \multicolumn{10}{|l|}{$\mathrm{A} 4$} \\
\hline Goal & & & & & & & & & \\
\hline
\end{tabular}

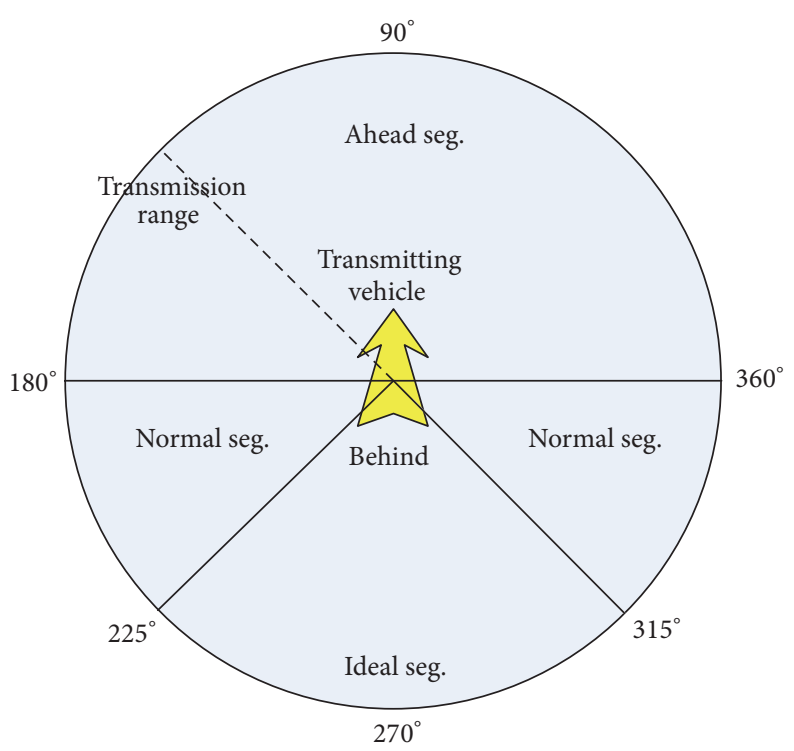

FIGURE 3: Wagon wheel: segmentation of a vehicle's transmission range.

TABLE 5: Summary of criteria for NFV selection.

\begin{tabular}{ll}
\hline Criteria & Explanation \\
\hline Direction & $\begin{array}{l}\text { Direction of R vehicle with respect to S/T vehicle, that } \\
\text { is, in same or opposite direction }\end{array}$ \\
Position & $\begin{array}{l}\text { Position of R vehicle with respect to the } \mathrm{S} / \mathrm{T} \text { vehicle, } \\
\text { that is, in ideal, normal or ahead segment }\end{array}$ \\
Distance & $\begin{array}{l}\text { Distance of the R vehicle from } \mathrm{S} / \mathrm{T} \text { vehicle, that is, } \\
\text { farthest or others }\end{array}$ \\
\hline
\end{tabular}

(iii) Distance. In the proposed method, the farthest vehicle inside transmission range is assigned the highest forwarding probability. Vehicles at extreme distance from transmitting as well as source vehicle (the border vehicles) are ideal to be the next forwarders.
Thus, the NFV selection decision is based on these parameters and mathematically it is represented in

$$
\text { NFV (Selection) }=\sum_{i \in P} P i
$$

where $i$ is the direction, position, or distance parameter and $P$ is the respective probability.

The wagon wheel as shown in Figure 3 is a circular transmission area of a vehicle where it can directly communicate with neighbor vehicles. It is mainly divided into two distinct segments: behind and ahead. The behind segment is a more important section of transmission range than the ahead segment during data dissemination process after an emergency event. It is further divided in two segments: ideal and normal. Ideal segment has the highest priority as compared to the normal and ahead segments. The vehicle(s) inside 


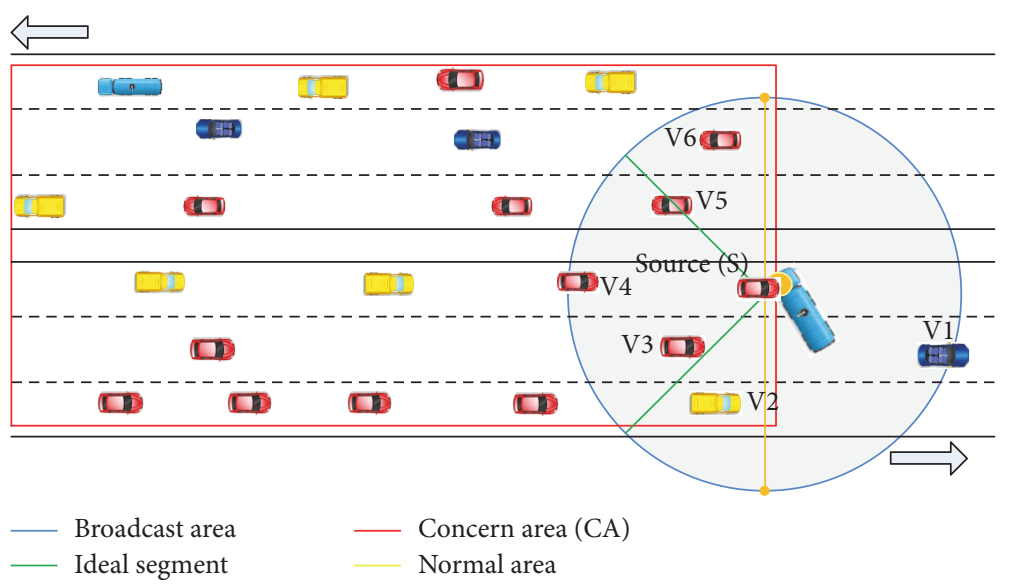

FIGURE 4: Highway scenario showing a data dissemination process after an accident.

ideal segment are best options to be the next forwarders of a data packet. In case no vehicle exists inside the ideal segment then vehicle(s) inside a normal segment will be preferred to be the next forwarders in order to retain the data dissemination process. Limited number of vehicle(s) selected as the $\mathrm{NFV}(\mathrm{s})$ are sufficient to distribute the information in the concern area of a network.

\section{NFV Selection Using ANP Model}

The performance in VANETs directly depends on the selection of next forwarder vehicle; therefore it is significant to select the optimum vehicle to effectively use limited bandwidth and minimize the redundant messages thus improving overall network performance. The selection of NFV is based on certain parameters such as direction, position, and distance which makes it a multicriteria decision problem. To select the optimum vehicle as NFV, in this paper we have used ANP method as a multicriteria decision tool. The interdependencies of alternatives (vehicles) are not considered in this analysis since the data dissemination process uses the receiver-based approach where each alternative has to calculate its probability independently. Even in this case, the priorities can be obtained with ANP to help decision maker. These priorities achieved form ANP method can be used directly in programmed model to implement the proposed method.

5.1. Case Study. In this case study, a3-lane bidirectional highway scenario with large number of vehicles (traffic) experiencing an event (accident) is considered to determine the most suitable vehicle as NFV inside the transmission range. The selected vehicle will distribute emergency warning messages (data packet) during the data dissemination process in VANETs, as shown in Figure 4.

A vehicle labeled $S$ (source) experiences an accident on highway and initiates data dissemination by flooding the emergency message (data packet) to all neighbor vehicles inside the concern area as shown in Figure 4. The data packet transmitted by $\mathrm{S}$ vehicle is received by $\mathrm{R}$ vehicles (in this case
V1-to-V6) inside its transmission range. After receiving the data packet, each $\mathrm{R}$ vehicle determines the following:

(i) Is data packet received for the first time? If yes, then schedule the transmission of received data packet as a suitable contestant for NFV selection process. Otherwise cancel the transmission of already scheduled data packet and discard it.

(ii) Is $\mathrm{R}$ in the same direction to $\mathrm{S}$ vehicle? If not (in opposite direction) then do not participate in data dissemination process during dense traffic conditions. It will be included in data dissemination process only during sparse traffic condition to deal with network partition problem.

(iii) Is $\mathrm{R}$ in ideal segment, that is, right behind the $\mathrm{S}$ vehicle? If yes, then set the shortest waiting time and set the highest forwarding probability. Otherwise, set the higher waiting time and assign lower forwarding probability.

(iv) Is $\mathrm{R}$ the border vehicle, that is, farthest vehicle, from $\mathrm{S} / \mathrm{T}$ vehicle? If yes then it is more important to be selected as the NFV than the other nonborder vehicles.

As discussed earlier, six vehicles are located inside the transmission range of the $\mathrm{S}$ vehicle. One vehicle will be considered as the NFV while simultaneously other vehicles have to discard the received data packet. In the same way, data dissemination process will continue until the emergency message is distributed successfully among all vehicles inside the concern area. Thus, proposed method guarantees information distribution in VANETs through the most appropriate vehicle with least delay, lowest number of retransmissions, and lowest number of collisions.

5.2. Proposed ANP Model. The ANP method provides solution to the decision problems that can be modeled diagrammatically (termed as network). The network contains clusters containing different elements. The elements of one cluster 


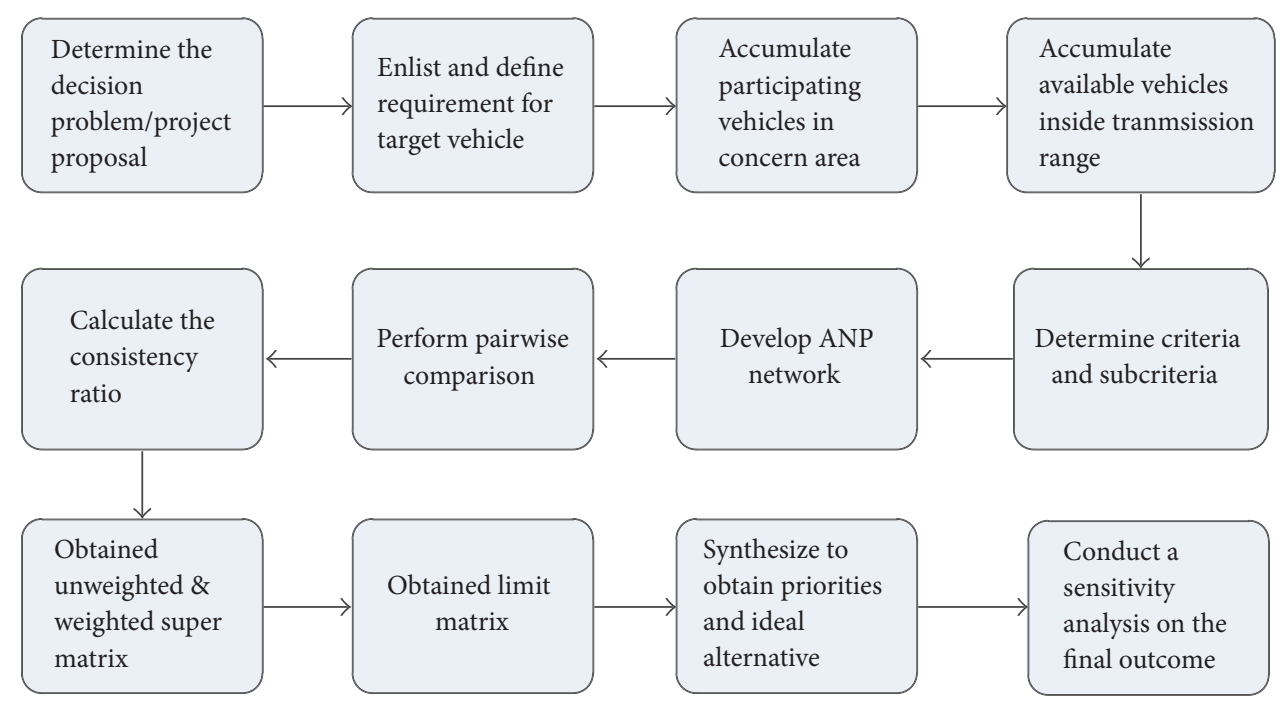

FIgURE 5: Steps involved in the proposed model based on ANP.

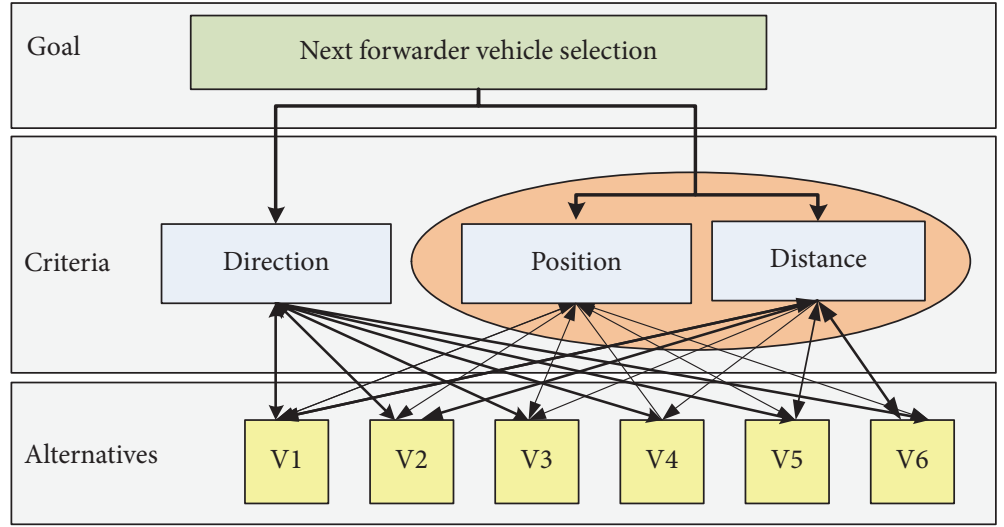

FIGURE 6: Construction and hierarchy of ANP model for NFV selection problem.

may connect with each other (inner dependence) and with elements of other clusters (outer dependence). The ANP network is concerned with every dependency that can affect an alternative. Section 3 covers the detailed steps involved in applying the ANP method to any complex decision problem. Figure 5 illustrates steps involved in the NFV selection problem based on ANP method.

Starting with ANP, at first we develop a hierarchy/ network by dividing the NFV selection problem in to goal, criteria, and alternatives. Goal is the objective that we want to achieve, criteria are the parameters considered for selection process, and alternatives are the available options to achieve the goal as shown in Figure 6.

5.3. Pairwise Comparisons. After structuring the problem and arranging the NFV problem in hierarchical form, we have to establish the priorities through pairwise comparisons among the elements of criteria and alternatives. The pairwise comparison of elements can be achieved in terms of importance, that is, for comparing a pair of elements of criteria we have to establish a ratio of relative importance between that pair of elements. This ratio depends on some standard scale to represent the relationship between two comparing elements. In ANP method, we use a fundamental scale for judgments in verbal as well as in numeric form presented by Saaty $[31,32]$ shown in Table 2. This scale shows the importance of one element over the other. For example, the relative importance of position with respect to distance of a vehicle is considered using 9-point quantitative scale. The position of a vehicle (with respect to transmitting vehicle) is extremely more important, strongly, moderately, equally important, or even extremely less important than the distance of vehicle (from 
transmitting vehicle). In pairwise comparison, a reciprocal score is allocated to the reverse comparison of that pair as illustrated in equation (1) and Table 2. In similar fashion, all pairwise comparisons are achieved for all considered elements and an initial matrix of judgments is obtained as shown in matrix (7) where all the diagonal values are 1 as every element is as important as itself.

$$
\left(\begin{array}{cccc} 
& \text { Position } & \text { Distance } & \text { Direction } \\
\text { Position } & 1 & & \\
\text { Distance } & & 1 & 1 \\
\text { Direction } & & & 1
\end{array}\right) \text {. }
$$

Usually, before assessing the importance of criteria and its elements, we assess the preference for alternatives with respect to the criteria. Using this approach we will acquire better understanding of alternatives as our judgments regarding the importance of criteria are dependent on alternatives. We have considered 6 vehicles (V1 to V6) as alternatives and we need three set of pairwise comparisons which reveals how well V1 to V6 perform in terms of these three (position, distance, and direction) criteria. The corresponding matrix of judgments is obtained as shown in matrix (8).

$$
\left(\begin{array}{ccccccc} 
& \mathrm{V} 1 & \mathrm{~V} 2 & \mathrm{~V} 3 & \mathrm{~V} 4 & \mathrm{~V} 5 & \mathrm{~V} 6 \\
\mathrm{~V} 1 & 1 & & & & & \\
\mathrm{~V} 2 & & 1 & & & & \\
\mathrm{~V} 3 & & & 1 & & & \\
\mathrm{~V} 4 & & & & 1 & & \\
\mathrm{~V} 5 & & & & & 1 & \\
\mathrm{~V} 6 & & & & & & 1
\end{array}\right) .
$$

Generally, we obtain the pairwise comparison matrices in the form of square and reciprocal matrices as shown in equation (1).

The pairwise comparisons, made in this study, were the direct results obtained from the opinions of a team of more than ten national and international experts both from academia and research community from different domains. Experts selected for this study are dynamically involved in the development and improvement of various routing and data dissemination scheme used in Intelligent Transportation Systems (ITS), Vehicular Ad hoc Networks (VANETs), Wireless Sensor Networks (WSNs), and delay/disruption Tolerant Networks (DTN). These experts were involved in almost every phase of the study and contributed well in the construction of ANP model, hierarchy and its levels, criteria and corresponding clusters, and recognition of inner and outer dependencies among elements and clusters. They also provide opinions regarding relative importance among elements as well as clusters and preferences for alternatives during qualitative pairwise comparisons. The variations in experts' opinions were normalized through the concepts of geometric means and the relative consensus value for each element was obtained. Moreover, in this study, we have used one of the most common aggregation approaches for the group decisions, that is, aggregate the individual judgments (AIJ) [33], since experts are associated with different organizations. Also the aggregation of individual judgments was accomplished through geometric mean because it is compatible with priorities and judgments [34]. Consistency ratio obtained after pairwise comparison was less than 0.1 which reveals the acceptable consistency among experts' opinions.

In this study, three different parameters: direction, position, and distance of a vehicle, are considered as the elements of the criteria in $n \times n$ matrix. The relative weight of each element is shown in the form of $W_{i j}$, where " $i$ " represents row and " $j$ " is the column of the matrix, respectively. If $W_{i j}=W_{j i}=1$, then the relative importance of element $W_{i}$ and $W_{j}$ is equal and assigned 1 for the elements located at main diagonal as shown in matrix (7) and (8). Once the relative weights of elements are decided, the ANP process for NFV selection problem proceeds (step-wise) as follows: Matrix (9a) shows the comparisons of alternatives (vehicles: V1-to-V6) with respect to the element "position" of criteria cluster.

$$
\left(\begin{array}{cccccccc} 
& \mathrm{V} 1 & \mathrm{~V} 2 & \mathrm{~V} 3 & \mathrm{~V} 4 & \mathrm{~V} 5 & \mathrm{~V} 6 \\
\mathrm{~V} 1 & 1 & \frac{1}{3}=0.33 & \frac{1}{7}=0.14 & \frac{1}{7}=0.14 & \frac{1}{7}=0.14 & \frac{1}{3}=0.33 \\
\mathrm{~V} 2 & 3 & 1 & \frac{1}{3}=0.33 & \frac{1}{3}=0.33 & \frac{1}{3}=0.33 & 1 \\
\mathrm{~V} 3 & 7 & 3 & 1 & 1 & 1 & 3 \\
\mathrm{~V} 4 & 7 & 3 & 1 & 1 & 1 & 3 \\
\mathrm{~V} 5 & 7 & 3 & 1 & 1 & 1 & 3 \\
\mathrm{~V} 6 & 3 & 1 & \frac{1}{3}=0.33 & \frac{1}{3}=0.33 & \frac{1}{3}=0.33 & 1
\end{array}\right)
$$

Add the weights in each column of matrix (9a) in order to obtain the total weight of columns (as shown in (9b)) and divide each weight of the same column by the obtained total weight (shown in $(9 c)$ ) in order to accomplish the normalization process involved in the pairwise comparisons. 


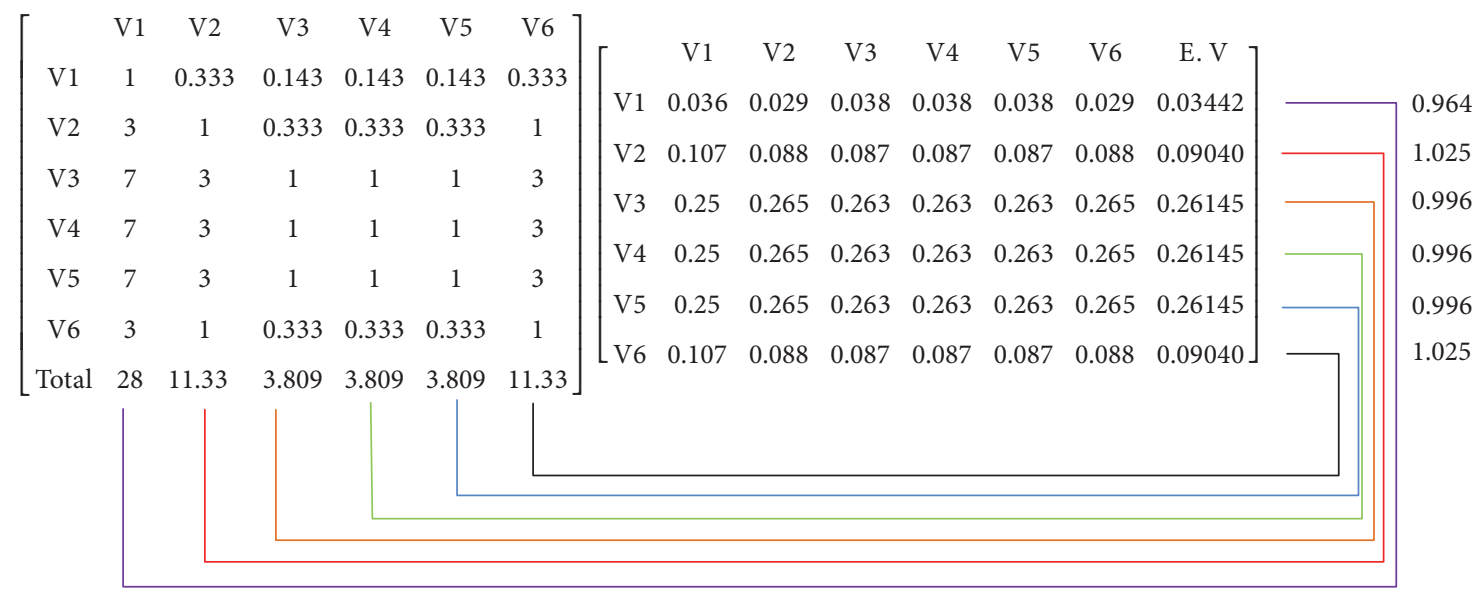

FIgURE 7: Graphical representation of Eigen value derivations.

$$
\left(\begin{array}{ccccccc} 
& \mathrm{V} 1 & \mathrm{~V} 2 & \mathrm{~V} 3 & \mathrm{~V} 4 & \mathrm{~V} 5 & \mathrm{~V} 6 \\
\mathrm{~V} 1 & 1 & 0.33 & 0.14 & 0.14 & 0.14 & 0.33 \\
\mathrm{~V} 2 & 3 & 1 & 0.33 & 0.33 & 0.33 & 1 \\
\mathrm{~V} 3 & 7 & 3 & 1 & 1 & 1 & 3 \\
\mathrm{~V} 4 & 7 & 3 & 1 & 1 & 1 & 3 \\
\mathrm{~V} 5 & 7 & 3 & 1 & 1 & 1 & 3 \\
\mathrm{~V} 6 & 3 & 1 & 0.33 & 0.33 & 0.33 & 1 \\
\text { Total } & 28 & 11.33 & 3.81 & 3.81 & 3.81 & 11.33
\end{array}\right)
$$

$$
\left(\begin{array}{ccccccc}
\mathrm{V} 1 & \mathrm{~V} 2 & \mathrm{~V} 3 & \mathrm{~V} 4 & \mathrm{~V} 5 & \mathrm{~V} 6 \\
\mathrm{~V} 1 & \frac{1}{28} & \frac{0.33}{11.33} & \frac{0.14}{3.81} & \frac{0.14}{3.81} & \frac{0.14}{3.81} & \frac{0.33}{11.33} \\
\mathrm{~V} 2 & \frac{3}{28} & \frac{1}{11.33} & \frac{0.33}{3.81} & \frac{0.33}{3.81} & \frac{0.33}{3.81} & \frac{1}{11.33} \\
\text { V3 } & \frac{7}{28} & \frac{3}{11.33} & \frac{1}{3.81} & \frac{1}{3.81} & \frac{1}{3.81} & \frac{3}{11.33} \\
\text { V4 } & \frac{7}{28} & \frac{3}{11.33} & \frac{1}{3.81} & \frac{1}{3.81} & \frac{1}{3.81} & \frac{3}{11.33} \\
\text { V5 } & \frac{7}{28} & \frac{3}{11.33} & \frac{1}{3.81} & \frac{1}{3.81} & \frac{1}{3.81} & \frac{3}{11.33} \\
\text { V6 } & \frac{3}{28} & \frac{1}{11.33} & \frac{0.33}{3.81} & \frac{0.33}{3.81} & \frac{0.33}{3.81} & \frac{1}{11.33}
\end{array}\right) .
$$

Matrix (9d) shows the normalized values obtained from the use of geometric mean (2) during the normalization process of matrix (9c) as follows:

$$
\left(\begin{array}{cccccccc} 
& \text { V1 } & \text { V2 } & \text { V3 } & \text { V4 } & \text { V5 } & \text { V6 } & \text { E.V } \\
\text { V1 } & 0.036 & 0.029 & 0.038 & 0.038 & 0.038 & 0.029 & 0.03442 \\
\text { V2 } & 0.107 & 0.088 & 0.087 & 0.087 & 0.087 & 0.088 & 0.09040 \\
\text { V3 } & 0.25 & 0.265 & 0.263 & 0.263 & 0.263 & 0.265 & 0.26145 \\
\text { V4 } & 0.25 & 0.265 & 0.263 & 0.263 & 0.263 & 0.265 & 0.26145 \\
\text { V5 } & 0.25 & 0.265 & 0.263 & 0.263 & 0.263 & 0.265 & 0.26145 \\
\text { V6 } & 0.107 & 0.088 & 0.087 & 0.087 & 0.087 & 0.088 & 0.09040
\end{array}\right) .
$$

Finally, to find the Eigenvalues, multiply total weight of each column of matrix (9b), that is, derived from pairwise comparisons, with the normalized value of each row in normalized matrix (9d). Figure 7 illustrates this procedure graphically.

$$
\left(\begin{array}{cccccccc} 
& \mathrm{V} 1 & \mathrm{~V} 2 & \mathrm{~V} 3 & \mathrm{~V} 4 & \mathrm{~V} 5 & \mathrm{~V} 6 & \mathrm{E} . \mathrm{V} \\
\mathrm{V} 1 & 1 & 0.333 & 0.1429 & 0.1429 & 0.1429 & 0.333 & 0.034 \\
\mathrm{~V} 2 & 3 & 1 & 0.333 & 0.333 & 0.333 & 1 & 0.09 \\
\mathrm{~V} 3 & 7 & 3 & 1 & 1 & 1 & 3 & 0.26 \\
\mathrm{~V} 4 & 7 & 3 & 1 & 1 & 1 & 3 & 0.26 \\
\mathrm{~V} 5 & 7 & 3 & 1 & 1 & 1 & 3 & 0.26 \\
\mathrm{~V} 6 & 3 & 1 & 0.333 & 0.333 & 0.333 & 1 & 0.09
\end{array}\right) .
$$

The consistency ratio (CR) for the matrix (9d) is calculated using (3), (4), and (5). The whole procedure is followed for obtaining the remaining matrices and relevant features. Matrix (10) represents the comparisons of alternatives (vehicles V1-to-V6) with respect to the criterion "distance."

$$
\left(\begin{array}{cccccccc} 
& \text { V1 } & \text { V2 } & \text { V3 } & \text { V4 } & \text { V5 } & \text { V6 } & \text { E.V } \\
\text { V1 } & 1 & 4 & 5 & 1 & 5 & 4 & 0.35 \\
\text { V2 } & 0.25 & 1 & 2 & 0.25 & 5 & 1 & 0.10 \\
\text { V3 } & 0.2 & 0.5 & 1 & 0.25 & 1 & 0.5 & 0.06 \\
\text { V4 } & 1 & 4 & 4 & 1 & 4 & 3 & 0.31 \\
\text { V5 } & 0.2 & 0.5 & 1 & 0.25 & 1 & 0.5 & 0.06 \\
\text { V6 } & 0.25 & 1 & 2 & 0.333 & 2 & 1 & 0.11
\end{array}\right) .
$$


The comparisons of alternatives (vehicles V1-to-V6) with respect to the criterion "direction" are represented in matrix (11).

$$
\left(\begin{array}{cccccccc} 
& \text { V1 } & \text { V2 } & \text { V3 } & \text { V4 } & \text { V5 } & \text { V6 } & \text { E.V } \\
\text { V1 } & 0.227 & 0.227 & 0.227 & 0.227 & 0.227 & 0.227 & 0.23 \\
\text { V2 } & 0.227 & 0.227 & 0.227 & 0.227 & 0.227 & 0.227 & 0.23 \\
\text { V3 } & 0.227 & 0.227 & 0.227 & 0.227 & 0.227 & 0.227 & 0.23 \\
\text { V4 } & 0.227 & 0.227 & 0.227 & 0.227 & 0.227 & 0.227 & 0.23 \\
\text { V5 } & 0.045 & 0.045 & 0.045 & 0.045 & 0.045 & 0.045 & 0.05 \\
\text { V6 } & 0.045 & 0.045 & 0.045 & 0.045 & 0.045 & 0.045 & 0.05
\end{array}\right) .
$$

Matrix (12) shows the comparisons of goal (NFV selection) with respect to the alternative cluster.

$$
\left(\begin{array}{cccc} 
& \text { Position } & \text { Distance } & \text { E.V } \\
\text { Position } & 1 & 7 & 0.875 \\
\text { Distance } & 0.1429 & 1 & 0.125
\end{array}\right) \text {. }
$$

The comparisons of $\mathrm{V} 1$ alternative with respect to elements in criteria 2 cluster are shown in matrix (13).

$$
\left(\begin{array}{cccc} 
& \text { Position } & \text { Distance } & \text { E.V } \\
\text { Position } & 1 & 5 & 0.833 \\
\text { Distance } & 0.2 & 1 & 0.167
\end{array}\right) \text {. }
$$

Similarly comparisons of alternatives "V2, V3, V4, V5, and V6" with respect to elements in "Criteria 2" cluster provide the similar weights as mentioned in matrix (13).

5.4. Unweighted and Weighted Supermatrix. The unweighted supermatrix is derived from all pairwise comparisons, where Eigen vector of each comparison is shown in their respective columns as shown in Table 6. As the obtained unweighted supermatrix is nonstochastic with respect to columns (i.e., sum of column weights $>1$ ), therefore, to make it column stochastic the cluster weight matrix (as shown in Table 7) is multiplied with unweighted supermatrix in order to transform into weighted supermatrix as shown in Table 8 . The cluster matrix reveals the impact of the criteria cluster on the alternatives. In our study, it seems that both clusters present equal impact on the alternatives, but as cluster 1 has only one element while cluster 2 is composed of two elements, thus we say that element of cluster 1 is influenced twice more than the elements of cluster 2 .

5.5. Limit Supermatrix. The limit supermatrix is obtained from the weighted supermatrix to get the stable values. The weighted supermatrix is raised up to a number of high powers until the convergence occurs to have same values in corresponding rows as shown in Table 9. According to the limit supermatrix, vehicle V4 (alternative) has highest priority and is selected as the next forwarder vehicle to disseminate the received data packet further inside a concern area. Limit supermatrix is basically the resultant matrix, which concludes

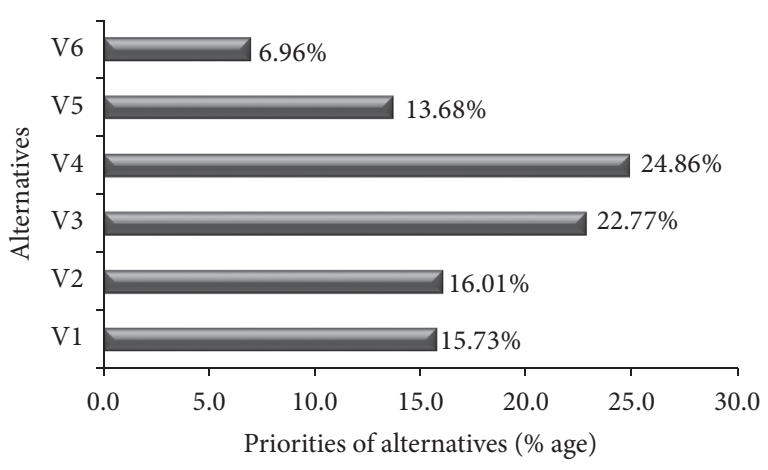

FIGURE 8: Percentage of priorities for alternatives of ANP model.

the overall process of ANP model. Moreover, it shows the alternatives ranking and is helpful in finding the criteria ranking.

The overall ranking of alternatives is shown in Figure 8, which shows the priorities of all alternatives and the one with maximum weight is selected as the most suitable vehicle for the highway scenario (Figure 4). Furthermore, vehicle V4 has the maximum weight followed by V3, V2, V1, V5, and V6, respectively. As V6 acquired the lowest possible priority, thus, it is the least preferred vehicle for data packet forwarding especially in dense traffic conditions. Moreover, as the priorities of V4 and V3 are very close to each other, we have to perform sensitivity analysis to confirm whether changes in weights of elements affect the priorities and overall ranking of alternatives.

5.6. Sensitivity Analysis. The sensitivity analysis is important for analyzing the stability of alternatives ranking but it is not mandatory. The sensitivity analysis for all three elements in criteria cluster for direction, position, and distance is carried out as shown in Figure 9. From previous sections (Sections 4 and 5), the "direction" of $\mathrm{R}$ vehicle with respect to $\mathrm{T}$ vehicle with highest weight was identified. In case we reduce the importance level of the direction of $\mathrm{R}$ vehicles, then the impact on ranking of alternatives can be examined in Figure 9(a). It discloses that ranking of the alternatives is altered and V5 is at second place instead of fifth besides the fact that ranking of V4 still has the highest forwarding priority. Similarly, by disregarding the importance of elements "position" and "direction," respectively, we will obtain the new ranking of vehicles shown in Figures 9(b) and 9(c).

For academia personnel, researchers, policy makers, and the industrialist working with Intelligent Transportation Systems (ITS) and its various aspects, this study presents useful approach of decision making process. Through this study, we have tried to illustrate that ANP method used for complex multicriteria decision problems presents important practical advantages by structuring the complete decision problem and showing the importance of various criteria all along with the feedback and dependencies. Furthermore, it helps out the decision makers in deciding whether their intuition was correct. This study discloses that the complex decision projects of different scale dimensions and sizes can be 
TABLE 6: Unweighted supermatrix.

\begin{tabular}{|c|c|c|c|c|c|c|c|c|c|}
\hline \multirow{2}{*}{ Cluster node labels } & \multicolumn{6}{|c|}{ Alternatives (available vehicles) } & \multicolumn{3}{|c|}{ Criteria (parameters) } \\
\hline & V1 & $\mathrm{V} 2$ & V3 & V4 & V5 & V6 & Direction & Position & Distance \\
\hline \multicolumn{10}{|l|}{ Alternatives } \\
\hline V1 & 0 & 0 & 0 & 0 & 0 & 0 & 0.22727 & 0.03444 & 0.35132 \\
\hline $\mathrm{V} 2$ & 0 & 0 & 0 & 0 & 0 & 0 & 0.22727 & 0.09090 & 0.10326 \\
\hline V3 & 0 & 0 & 0 & 0 & 0 & 0 & 0.22727 & 0.26125 & 0.06242 \\
\hline $\mathrm{V} 4$ & 0 & 0 & 0 & 0 & 0 & 0 & 0.22727 & 0.26125 & 0.31302 \\
\hline V5 & 0 & 0 & 0 & 0 & 0 & 0 & 0.04546 & 0.26125 & 0.06242 \\
\hline V6 & 0 & 0 & 0 & 0 & 0 & 0 & 0.04546 & 0.09090 & 0.10756 \\
\hline \multicolumn{10}{|l|}{ Criteria } \\
\hline Direction & 1 & 1 & 1 & 1 & 1 & 1 & 0 & 0 & 0 \\
\hline Position & 0.8333 & 0.8333 & 0.8333 & 0.8333 & 0.8333 & 0.8333 & 0 & 0 & 0 \\
\hline Distance & 0.1667 & 0.1667 & 0.1667 & 0.1667 & 0.1667 & 0.1667 & 0 & 0 & 0 \\
\hline
\end{tabular}

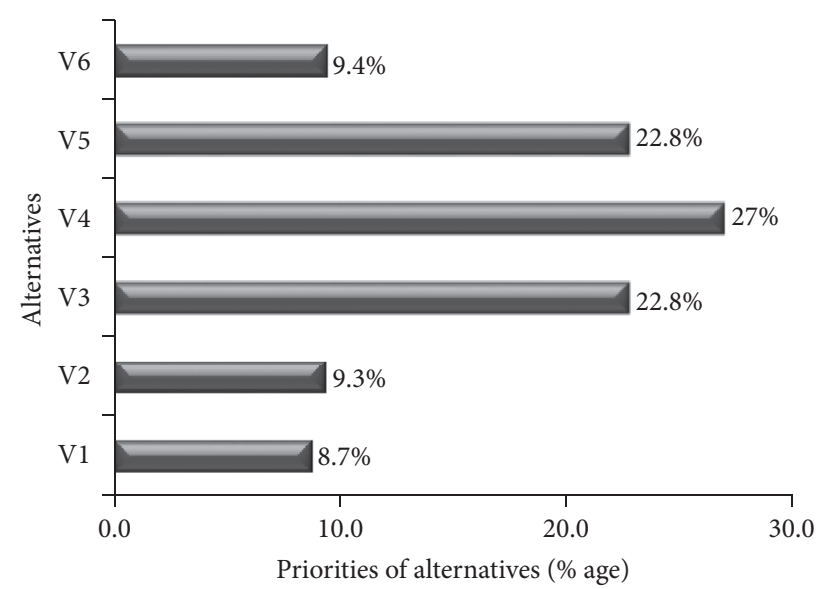

(a) Influence of criterion "Direction" on "Alternatives"

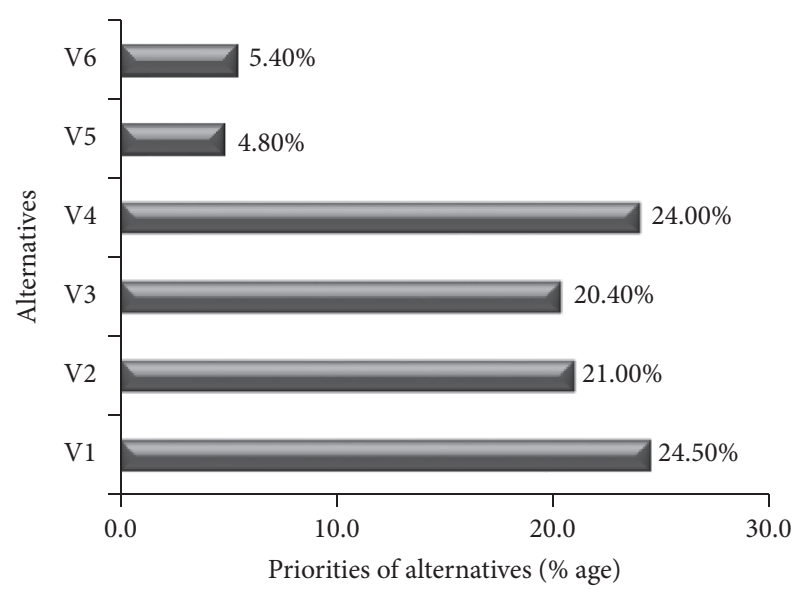

(b) Influence of criterion "Position" on "alternatives"

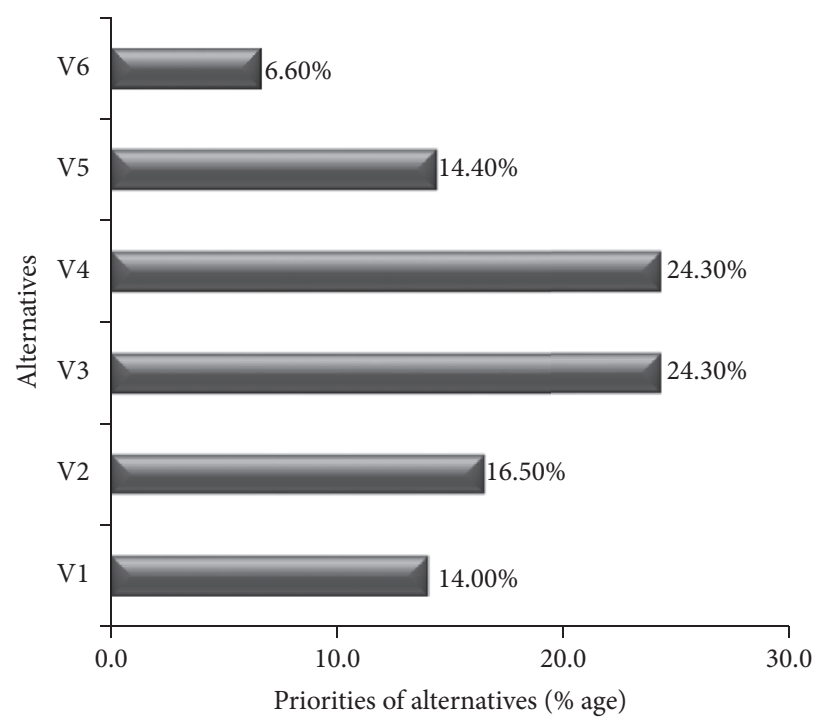

(c) Influence of criterion "Distance" on "alternatives"

FIGURE 9: Sensitivity analysis for the criterion: (a) direction, (b) position, and (c) distance. 
TABLE 7: Cluster weight matrix.

\begin{tabular}{lcccc}
\hline Cluster Label & Alternative & Criteria 1 & Criteria 2 & Goal \\
\hline Alternative & 0 & 1 & 1 & 0 \\
Criteria 1 & 0.5 & 0 & 0 & 0.5 \\
Criteria 2 & 0.5 & 0 & 0 & 0.5 \\
Goal & 0 & 0 & 0 & 0 \\
\hline
\end{tabular}

evaluated using ANP method. Major drawback of this study is the consideration of very limited alternatives to be evaluated, as ANP method supports limited alternatives [26, 31].

\section{Results and Discussions}

The performance of the proposed method is further evaluated using the vehicular network simulation framework VEINS [15] which integrates the network simulator OMNET++ [35] and the Simulator for Urban MObility (SUMO) [36]. The evaluation is performed in terms of reachability, latency, total transmitted data packets, number of collisions, and the duplicate data packets. Furthermore, the proposed method is compared with existing well-known data dissemination protocols such as Simple Flooding, AID, and DBRS.

In VANETs, data dissemination is a broadcast-oriented process which may lead the network to face the broadcast storm problem and inefficient use of network resources such as bandwidth. Therefore, to avoid the broadcast storm and utilize the network resources efficiently, next forwarder vehicles (NFVs) are selected to forward the received data packet to all intended vehicles of network. The NFV selection is based on certain parameters such as direction, distance, and position information of vehicles. The NFV selection based on the aforementioned parameters of vehicle certainly increases the efficiency and robustness of a data dissemination protocol to its maximum potential.

To evaluate the performance of the proposed method against existing data dissemination protocols, the following evaluation metrics were considered.

(i) Reachability. This metric defines the ability to be aware of an event. In VANETs it is the ratio of vehicles successfully receiving the broadcast data packet to the total number of reachable vehicles [37]. It is used to evaluate the transmitted data packet through the entire vehicular network. Its high value represents better broadcast efficiency of a data dissemination protocol. Sometimes, it is also measured in terms of Broadcast Coverage Ratio (BCR) or Broadcast Delivery Ratio (BDR) metrics.

(ii) Latency. It presents how much time is spent by a broadcast data packet to traverse through the network, from its source vehicle to the designated vehicle in VANETs. It is an important metric to evaluate the efficiency of data dissemination protocol designed for delay sensitive safety applications such as an emergency or traffic jam. Low latency is essential for such data dissemination protocols. (iii) Number of Collisions. It shows the average number of data packets collisions at MAC layer for all vehicles. High collision rate during dense traffic conditions may lead the network to meet the broadcast storm problem because many vehicles rebroadcast the same data packet in same vicinity. Thus least number of collisions is required for efficient and reliable data dissemination. Sometimes, it is also measured in terms of Collision Ratio (CR) or Packet Loss Ratio (PLR) metrics.

(iv) Total Transmitted Data Packets. This metric corresponds to the total number of data packets transmitted by all vehicles in a network during data dissemination process. Large number of transmissions occurs due to redundant data packets and may incur the broadcast storm problem.

(v) Duplicate Data Packets. This metric shows average number of replicated data packets received by each vehicle inside the concern area of a vehicular network. It is important to analyze the robustness of a data dissemination protocol.

6.1. Simulation Setup. In this scenario, we considered a 10kilometer long highway with vehicles moving in two opposite directions. Vehicle flow is generated on each edge of the highway that inserts different traffic flow at constant rate starting from 800 to 2000 vehicles/hour. For more realistic vehicular scenario, the mobility of inserted vehicles is set different to ensure the overtaking of vehicles. These vehicles are capable of reaching a maximum speed of $33 \mathrm{~m} / \mathrm{s}, 26 \mathrm{~m} / \mathrm{s}$, and $20 \mathrm{~m} / \mathrm{s}$. Once the simulation become stable, a vehicle stops abruptly at highway describing an emergency. It initiates data dissemination process by generating and broadcasting a data packet inside the concern area of network. Our intention is to alert the vehicular traffic about emergency, approaching towards the place of emergency, through multihop communication. Other important parameters including bit rate, data packet size, and transmission range are presented in Table 10.

6.2. Performance Evaluation of the Proposed Method. The proposed method is compared with other well-known data dissemination protocols in terms of reachability, latency, saved broadcast, and total number of transmitted data packets. All protocols are evaluated under highway scenario with varying traffic conditions as shown in Figures 10-14. The results reveal that the proposed method outperforms the existing data dissemination protocols in terms of efficient and reliable data dissemination in vehicular environment.

In Figure 10, the reachability in terms of successful data dissemination is determined at highway scenario under varying traffic conditions. The proposed method shows better performance by acquiring maximum reachability especially at dense traffic conditions. It is worth noticing that the proposed method presents closer reachability results to the Flooding protocol. However, Flooding protocol turns out in high number of transmissions with growing vehicular traffic and leads to the broadcast storm problem.

The proposed method is evaluated for latency under varying traffic conditions as shown in Figure 11. It shows that 
TABLE 8: Weighted supermatrix.

\begin{tabular}{|c|c|c|c|c|c|c|c|c|c|}
\hline \multirow{2}{*}{ Cluster node labels } & \multicolumn{6}{|c|}{ Alternatives (available vehicles) } & \multicolumn{3}{|c|}{ Criteria (parameters) } \\
\hline & V1 & $\mathrm{V} 2$ & V3 & $\mathrm{V} 4$ & V5 & V6 & Direction & Position & Distance \\
\hline \multicolumn{10}{|l|}{ Alternatives } \\
\hline V1 & 0 & 0 & 0 & 0 & 0 & 0 & 0.22727 & 0.03444 & 0.35132 \\
\hline $\mathrm{V} 2$ & 0 & 0 & 0 & 0 & 0 & 0 & 0.22727 & 0.0909 & 0.10326 \\
\hline V3 & 0 & 0 & 0 & 0 & 0 & 0 & 0.22727 & 0.26125 & 0.06242 \\
\hline V4 & 0 & 0 & 0 & 0 & 0 & 0 & 0.22727 & 0.26125 & 0.31302 \\
\hline V5 & 0 & 0 & 0 & 0 & 0 & 0 & 0.04546 & 0.26125 & 0.06242 \\
\hline V6 & 0 & 0 & 0 & 0 & 0 & 0 & 0.04546 & 0.0909 & 0.10756 \\
\hline \multicolumn{10}{|l|}{ Criteria } \\
\hline Direction & 0.5 & 0.5 & 0.5 & 0.5 & 0.5 & 0.5 & 0 & 0 & 0 \\
\hline Position & 0.4167 & 0.4167 & 0.4167 & 0.4167 & 0.4167 & 0.4167 & 0 & 0 & 0 \\
\hline Distance & 0.0833 & 0.0833 & 0.0833 & 0.0833 & 0.0833 & 0.0833 & 0 & 0 & 0 \\
\hline
\end{tabular}

TABle 9: Limit supermatrix.

\begin{tabular}{|c|c|c|c|c|c|c|c|c|c|}
\hline \multirow{2}{*}{ Cluster/node labels } & \multicolumn{6}{|c|}{ Alternatives (available vehicles) } & \multicolumn{3}{|c|}{ Criteria (parameters) } \\
\hline & V1 & $\mathrm{V} 2$ & V3 & V4 & V5 & V6 & Direction & Position & Distance \\
\hline \multicolumn{10}{|l|}{ Alternatives } \\
\hline V1 & 0.07863 & 0.07863 & 0.07863 & 0.07863 & 0.07863 & 0.07863 & 0.07863 & 0.07863 & 0.07863 \\
\hline $\mathrm{V} 2$ & 0.08006 & 0.08006 & 0.08006 & 0.08006 & 0.08006 & 0.08006 & 0.08006 & 0.08006 & 0.08006 \\
\hline V3 & 0.11385 & 0.11385 & 0.11385 & 0.11385 & 0.11385 & 0.11385 & 0.11385 & 0.11385 & 0.11385 \\
\hline V4 & 0.12429 & 0.12429 & 0.12429 & 0.12429 & 0.12429 & 0.12429 & 0.12429 & 0.12429 & 0.12429 \\
\hline V5 & 0.06839 & 0.06839 & 0.06839 & 0.06839 & 0.06839 & 0.06839 & 0.06839 & 0.06839 & 0.06839 \\
\hline V6 & 0.03478 & 0.03478 & 0.03478 & 0.03478 & 0.03478 & 0.03478 & 0.03478 & 0.03478 & 0.03478 \\
\hline \multicolumn{10}{|l|}{ Criteria } \\
\hline Direction & 0.25 & 0.25 & 0.25 & 0.25 & 0.25 & 0.25 & 0.25 & 0.25 & 0.25 \\
\hline Position & 0.20833 & 0.20833 & 0.20833 & 0.20833 & 0.20833 & 0.20833 & 0.20833 & 0.20833 & 0.20833 \\
\hline Distance & 0.04167 & 0.04167 & 0.04167 & 0.04167 & 0.04167 & 0.04167 & 0.04167 & 0.04167 & 0.04167 \\
\hline
\end{tabular}

TABLE 10: Simulation parameters for highway scenario.

\begin{tabular}{lc}
\hline Parameter & Value \\
\hline Transmission power & $1.6 \mathrm{~mW}$ \\
Transmission range & $250 \mathrm{~m}$ \\
Bit rate & $18 \mathrm{Mbit} / \mathrm{s}$ \\
Concern area & $5 \mathrm{Km}$ \\
Data packet size & $2048 \mathrm{bytes}$ \\
Highway length & $10 \mathrm{Km}$ \\
Road direction/lanes & One-way/3 \\
\hline
\end{tabular}

AID protocol presents the highest latency due to the use of counter-base forwarding strategy. In AID, each vehicle waits and counts the number of received data packets sent by other vehicles of network. On the basis of data packet reception rate, it decides whether to retransmit the data packet further or let it for someone else. In proposed method, latency is inversely related to the traffic density. Thus growing density of vehicles inside the concern area results in decreasing latency as vehicles retransmit the data packet with lowest waiting time. DBRS protocol presents lower latency than the proposed method however it does not perform well in terms of reachability metric and offers lower reachability.
Similarly, Flooding offers the lowest latency in comparison with AID, DBRS, and the proposed method; however it causes maximum number of transmitted data packets and larger number of collisions incurring the broadcast storm problem. Although proposed method turns out in little extra latency at sparse traffic conditions, its latency decreases significantly with increasing vehicular traffic.

In Figure 12, the number of overall transmitted data packets during data dissemination process is shown for the proposed method and all other protocols. This metric analyzes the efficiency of data dissemination protocols in terms of mitigating the broadcast storm during dense traffic conditions. Hence a protocol with reduced number of transmitted data packets mitigates the broadcast storm efficiently. Figure 12 depicts that Flooding protocol produces highest number of transmitted data packets. In fact, Flooding protocol does not use any broadcast suppression mechanism to control and reduce the transmission rate. The proposed method shows acceptable overhead than Flooding protocol by an average of $55 \%$. Similarly, AID and DBRS protocols produce less number of transmitted data packets than the proposed method; however the proposed method maximizes the reachability by approximately $30 \%$ and $70 \%$ in comparison with AID and DBRS, respectively. 


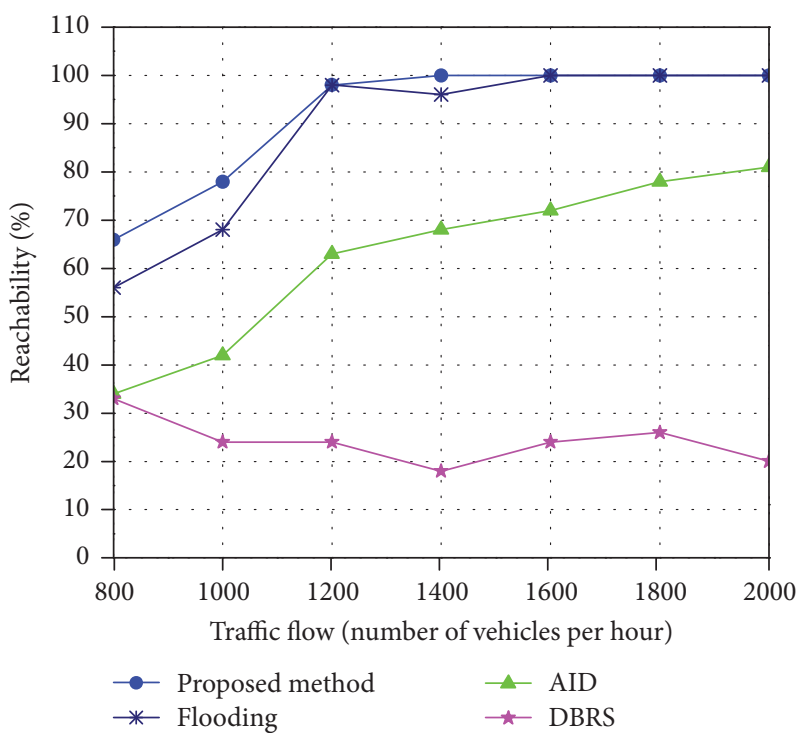

FIGURE 10: Reachability under varying traffic flow.

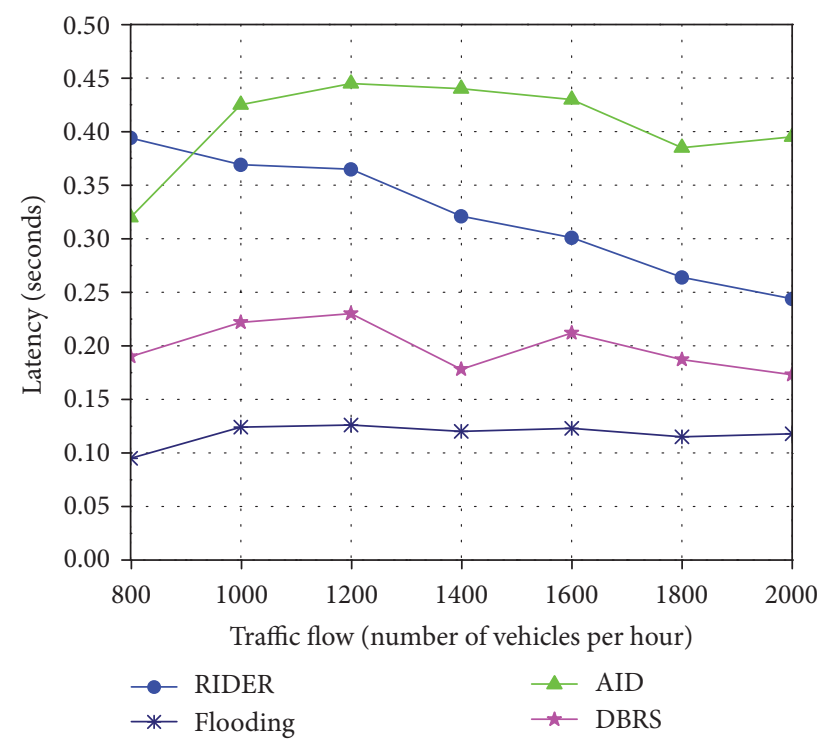

Figure 11: Latency with respect to Traffic flow.

Number of packets collisions at MAC layer evaluates the performance of data dissemination protocols in terms of failure rate particularly in dense traffic conditions. Figure 13 presents all results for average number of data packets collisions. Consequently, Flooding protocol shows the highest packet collision gradually increasing with increasing vehicular traffic. In Flooding protocol, each vehicle blindly retransmits the received data packet and accesses the bandwidth simultaneously. The proposed method presents closer results to AID and DBRS in terms of collision, that is, approximately one collision per vehicle.

Number of duplicate data packets analyzes the robustness of data dissemination protocol. Figure 14 depicts that each

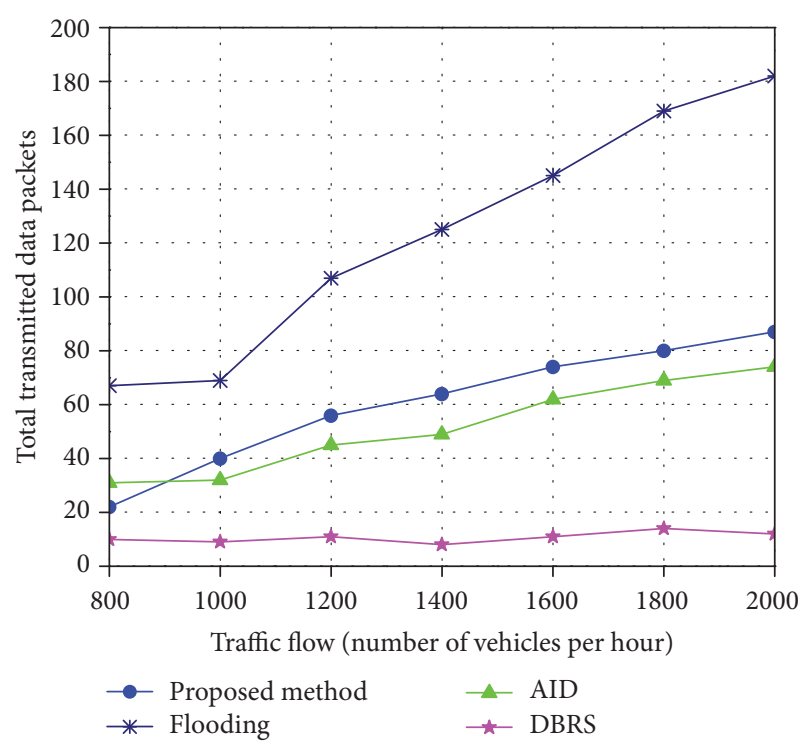

FIGURE 12: Total number of transmitted data packets.

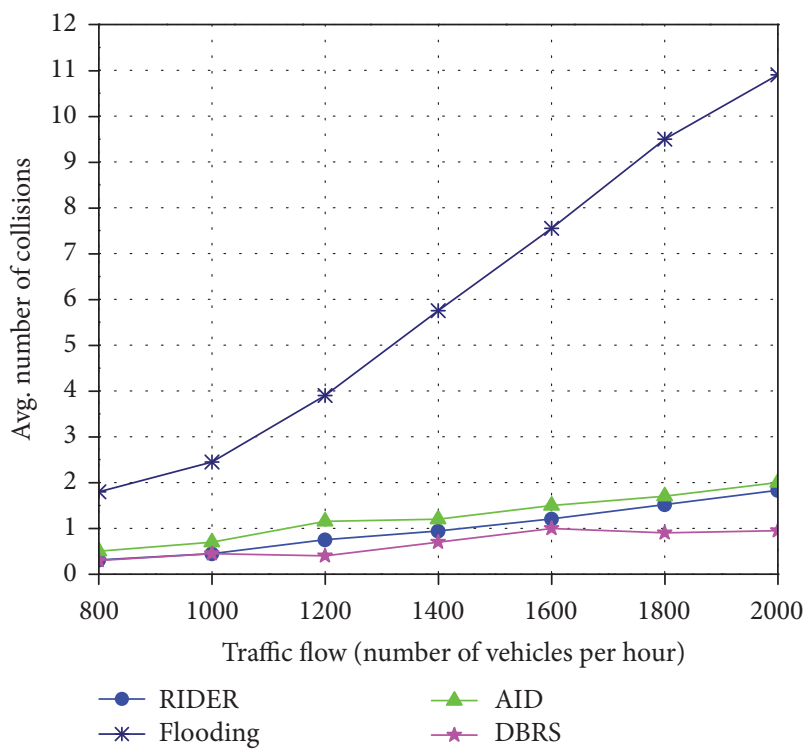

Figure 13: Number of Collisions with varying traffic flow.

vehicle in the AID, Flooding protocol, and the proposed method receives on average four to five duplicate packets during data dissemination process. The importance of duplicate data packets reception can be directly revealed from the reachability as shown in Figure 10. It can be observed that the proposed method presents similar reception number of duplicate packets in comparison to Flooding protocol. However the proposed method outperforms the Flooding protocol in terms of reduced collisions and lower number of transmitted packets. DBRS protocol offers less number of duplicate packets and hence minimum reachability.

Finally, we conclude that the proposed method mitigates the broadcast storm efficiently in highway VANET scenario 


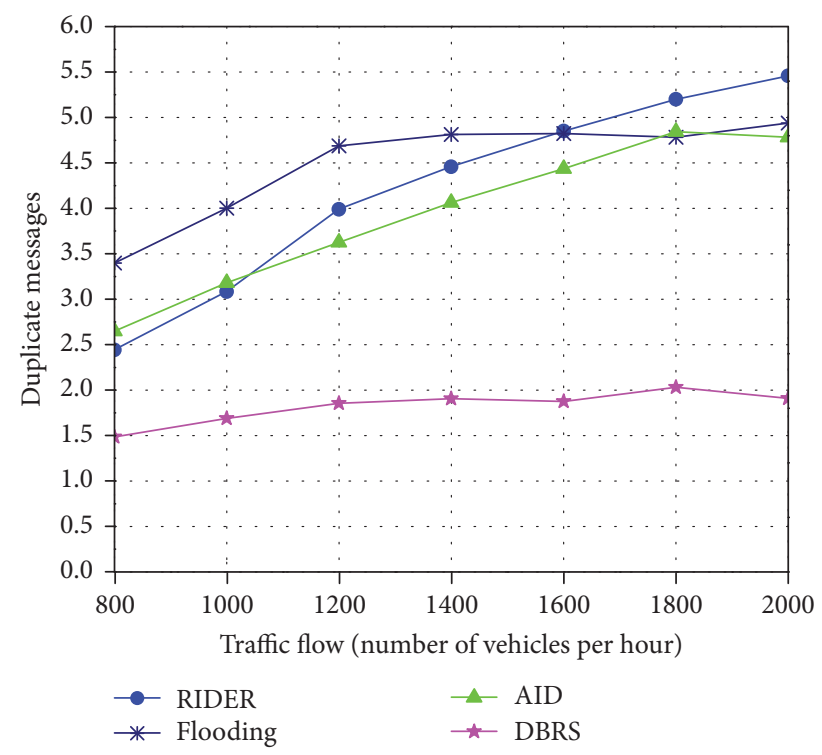

FIGURE 14: Duplicate messages with respect to traffic flow.

using the broadcast suppression technique. Moreover it delivers the data packet to all intended vehicles inside the concern area of a network with acceptable delay and network overhead.

\section{Conclusion and Future Directions}

In this paper, the optimum selection of next forwarder vehicle is considered for data dissemination in VANETs using multicriteria decision tool, namely, analytical network process. The selection of an appropriate vehicle as the NFV among a set of vehicles during data dissemination process certainly increases efficiency and robustness of a data dissemination protocol to its maximum potential. The NFV is selected on the basis of three parameters, direction, position, and distance, using ANP model. The proposed method is applied on highway scenario by considering a case study. The results obtained during this study demonstrate that vehicle(s) moving in similar direction to source/transmitting vehicle, positioned inside ideal segment of its transmission range along with furthest distance, will be selected as the NFV. In the case study, vehicle V4 is the most preferred while V6 is the least preferred option as NFV. In case, vehicle V4 does not forward the received data packet after being selected as NFV due to selfish behavior or leaving network before transmission; then vehicle V3 will transmit data packet just after completing the assigned waiting time. The stability of alternatives ranking is checked using sensitivity analysis to check the influence of the criteria on overall ranking of alternatives. By changing weight of any element in criteria the corresponding weights are changed accordingly. It is worth noticing that the overall ranking of the alternatives remains the same. Therefore, it is concluded that the alternative ranking is stable and optimum node is selected as NFV. Simulation results presented highlight that the proposed data dissemination scheme comparatively shows better results for different performance parameters. In future current work will be extended for more parameters and tested on different urban scenarios in VANETs.

\section{Conflicts of Interest}

The authors declare that there are no conflicts of interest regarding the publication of this paper.

\section{References}

[1] M. Van Eenennaam, W. K. Wolterink, G. Karagiannis, and G. Heijenk, "Exploring the solution space of beaconing in VANETs," in Proceedings of the 2009 IEEE Vehicular Networking Conference, VNC 2009, pp. 1-8, October 2009.

[2] K. Dar, M. Bakhouya, J. Gaber, M. Wack, and P. Lorenz, "Wireless communication technologies for ITS applications," IEEE Communications Magazine, vol. 48, no. 5, pp. 156-162, 2010.

[3] C. Sommer and F. Dressler, Vehicular Networking, Cambridge University Press, Cambridge, UK, 2014.

[4] J. Sousanis, "World vehicle population tops 1 billion units," Wards Auto, vol. 15, 2011, http://wardsauto.com/ar/world_vehicle_ population_110815.

[5] J. John Voelcker, "1.2 billion vehicles on world's roads now, 2 billion by 2035: report," Green Car Reports, vol. 7, no. 29, article 14, 2015.

[6] World Health Organization. Violence, Injury Prevention and World Health Organization, 2013. Global status report on road safety 2013: supporting a decade of action. World Health Organization.

[7] H. Hartenstein, VANET: Vehicular Applications and InterNetworking Technologies, vol. 1, Wiley, Chichester, UK, 2010.

[8] O. K. Tonguz, N. Wisitpongphan, and F. Bai, "DV-CAST: a distributed vehicular broadcast protocol for vehicular ad hoc networks," IEEE Wireless Communications, vol. 17, no. 2, pp. 47$57,2010$.

[9] Y.-S. Chen, Y.-W. Lin, and S.-L. Lee, "A mobicast routing protocol in vehicular ad-hoc networks," Mobile Networks and Applications, vol. 15, no. 1, pp. 20-35, 2010.

[10] Y.-S. Chen and Y.-W. Lin, "A mobicast routing protocol with carry-and-forward in vehicular ad hoc networks," International Journal of Communication Systems, vol. 27, no. 10, pp. 1416-1440, 2014.

[11] L. A. Villas, T. P. de Andrade, and N. L. da Fonseca, "An efficient and robust protocol to disseminate data in highway environments with different traffic conditions," in Proceedings of the 2014 IEEE Symposium on Computers and Communications (ISCC), pp. 1-6, IEEE, 2014.

[12] N. Wisitpongphan, O. K. Tonguz, J. S. Parikh, P. Mudalige, F. Bai, and V. Sadekar, "Broadcast storm mitigation techniques in vehicular ad hoc networks," IEEE Wireless Communications, vol. 14, no. 6, pp. 84-94, 2007.

[13] M. Bakhouya, J. Gaber, and P. Lorenz, "An adaptive approach for information dissemination in Vehicular Ad hoc Networks," Journal of Network and Computer Applications, vol. 34, no. 6, pp. 1971-1978, 2011.

[14] T. H. Kim, W. K. Hong, H. C. Kim, and Y. D. Lee, "An effective data dissemination in vehicular ad-hoc network," in Proceedings of the In International Conference on Information Networking, pp. 295-304, Springer, Berlin, Germany, January 2007. 
[15] C. Sommer, R. German, and F. Dressler, "Bidirectionally coupled network and road traffic simulation for improved IVC analysis," IEEE Transactions on Mobile Computing, vol. 10, no. 1, pp. 3-15, 2011.

[16] L. A. Villas, A. Boukerche, G. Maia, R. W. Pazzi, and A. A. F. Loureiro, "DRIVE: an efficient and robust data dissemination protocol for highway and urban vehicular ad hoc networks," Computer Networks, vol. 75, pp. 381-394, 2014.

[17] M. Durresi, A. Durresi, and L. Barolli, "Emergency broadcast protocol for inter-vehicle communications," in Proceedings of the 11th International Conference on Parallel and Distributed Systems (ICPADS'05), vol. 2, pp. 402-406, IEEE, July 2005.

[18] S. Panichpapiboon and G. Ferrari, "Irresponsible forwarding," in Proceedings of the 2008 8th International Conference on ITS Telecommunications (ITST), pp. 311-316, Phuket, Thailand, October 2008.

[19] R. S. Schwartz, R. R. R. Barbosa, N. Meratnia, G. Heijenk, and H. Scholten, "A directional data dissemination protocol for vehicular environments," Computer Communications, vol. 34, no. 17, pp. 2057-2071, 2011.

[20] J. Wallenius, J. S. Dyer, P. C. Fishburn, R. E. Steuer, S. Zionts, and K. Deb, "Multiple criteria decision making, multi-attribute utility theory: recent accomplishments and what lies ahead," Management Science, vol. 54, no. 7, pp. 1336-1349, 2008.

[21] H. Farman, H. Javed, B. Jan et al., "Analytical network process based optimum cluster head selection in wireless sensor network," PLOS ONE, vol. 12, no. 7, 2017.

[22] B. Roy, "The optimisation problem formulation: Criticism and overstepping," Journal of the Operational Research Society, vol. 32, no. 6, pp. 427-436, 1981.

[23] B. E. Costa and A. Carlos, "Les problématiques de l'aide à la décision: vers l'enrichissement de la trilogie choix-trirangement," Revue Française D'automatique, D'informatique Et De Recherche Opérationnelle, vol. 30, no. 2, pp. 191-216, 1996.

[24] R. L. Keeney and R. L. Keeney, Value-Focused Thinking: A Path to Creative Decisionmaking, Harvard University Press, Cambridge, Mass, USA, 2009.

[25] E. Triantaphyllou, "Multi-criteria decision making methods," in Multi-criteria Decision Making Methods: A Comparative Study, vol. 44 of Applied Optimization, pp. 5-21, Springer US, Boston, MA, 2000.

[26] T. L. Saaty, Theory and Applications of the Analytic Network Process, RWS Publications, Pittsburgh, Penn, USA, 2005.

[27] S. Thomas, The Analytic Hierarchy Process: Planning, Priority Setting, Resource Allocation, University of Pittsburgh, Pittsburgh, Penn, USA, 1980.

[28] H. Lee, S. Lee, and Y. Park, "Selection of technology acquisition mode using the analytic network process," Mathematical and Computer Modelling, vol. 49, no. 5-6, pp. 1274-1282, 2009.

[29] B. Pang and S. Bai, "An integrated fuzzy synthetic evaluation approach for supplier selection based on analytic network process," Journal of Intelligent Manufacturing, vol. 24, no. 1, pp. 163-174, 2013.

[30] Y.-C. Hu, "Analytic network process for pattern classification problems using genetic algorithms," Information Sciences, vol. 180, no. 13, pp. 2528-2539, 2010.

[31] T. L. Saaty, Theory and Applications of the Analytic Network Process: Decision Making with Benefits, Opportunities, Costs, and Risks, RWS publications, Pittsburgh, Penn, USA, 2005.

[32] T. L. Saaty, Multicriteria Decision Making: The Analytic Hierarchy Process, University of Pittsburgh Press, Pittsburgh, Penn, USA, 1998.
[33] J. Aznar, J. Ferrís-Oñate, and F. Guijarro, "An ANP framework for property pricing combining quantitative and qualitative attributes," Journal of the Operational Research Society, vol. 61, no. 5, pp. 740-755, 2010.

[34] R. Aull-Hyde, S. Erdogan, and J. M. Duke, "An experiment on the consistency of aggregated comparison matrices in AHP," European Journal of Operational Research, vol. 171, no. 1, pp. 290-295, 2006.

[35] A. s. Varga and R. Hornig, "An overview of the OMNeT++ simulation environment," in Proceedings of the 1st International Conference on Simulation Tools and Techniques for Communications, Networks and Systems \& Workshops, Institute for Computer Sciences, Social-Informatics and Telecommunications Engineering, 2008.

[36] M. Behrisch, L. Bieker, J. Erdmann, and D. Krajzewicz, "SUMOsimulation of urban mobility: an overview," in Proceedings of the Third International Conference on Advances in System Simulation, SIMUL 2011, ThinkMind, 2011.

[37] S. Panichpapiboon and W. Pattara-Atikom, "A review of information dissemination protocols for vehicular ad Hoc networks," IEEE Communications Surveys \& Tutorials, vol. 14, no. 3, pp. 784-798, 2012. 


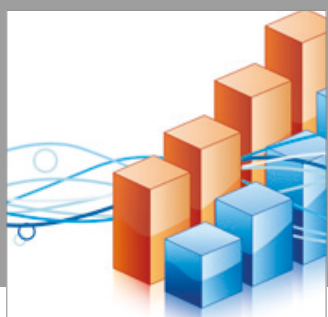

Advances in

Operations Research

vatersals

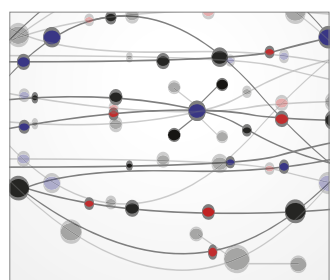

\section{The Scientific} World Journal
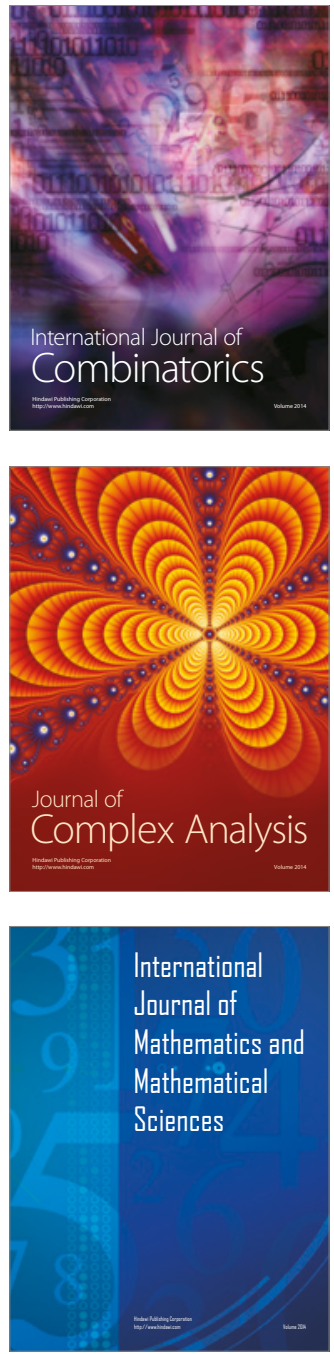
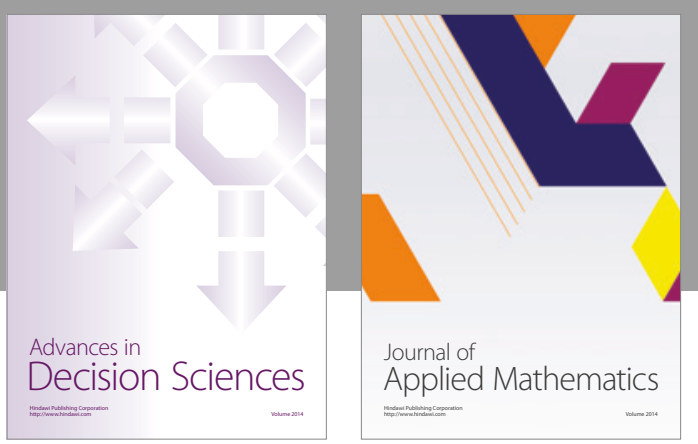

Algebra

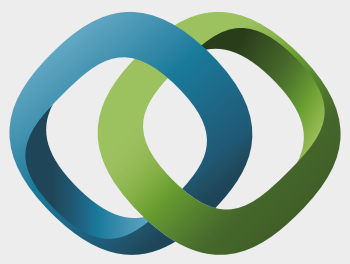

\section{Hindawi}

Submit your manuscripts at

https://www.hindawi.com
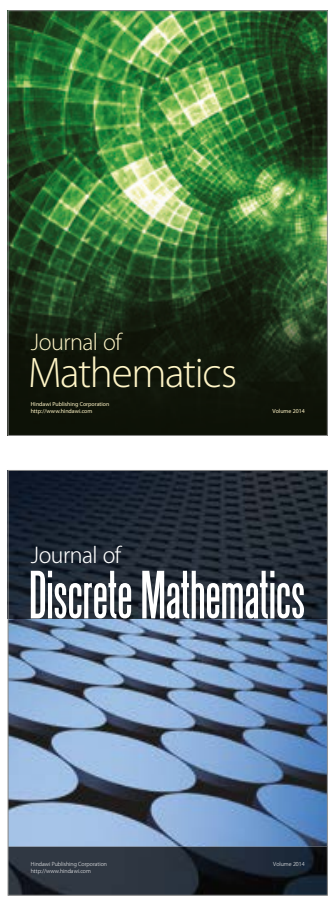

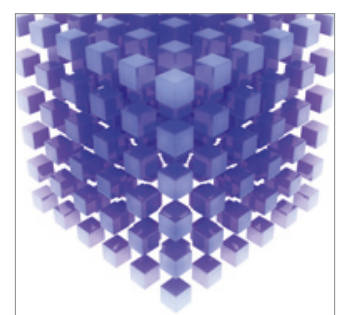

Mathematical Problems in Engineering
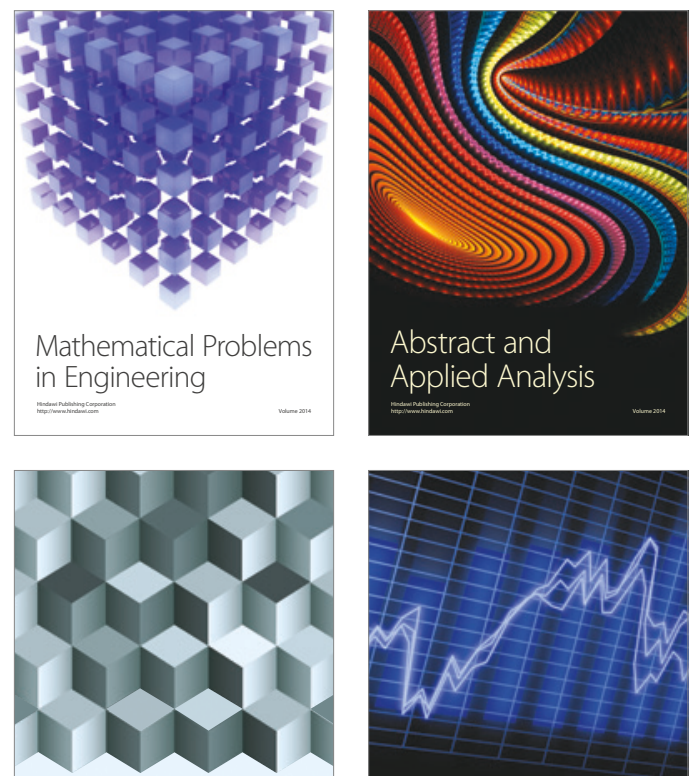

Journal of

Function Spaces

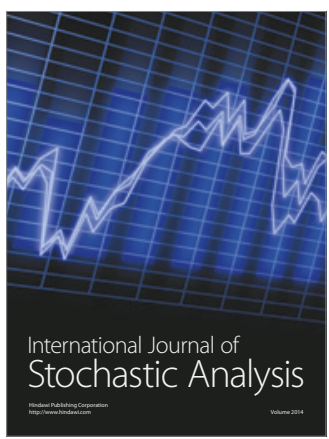

Probability and Statistics
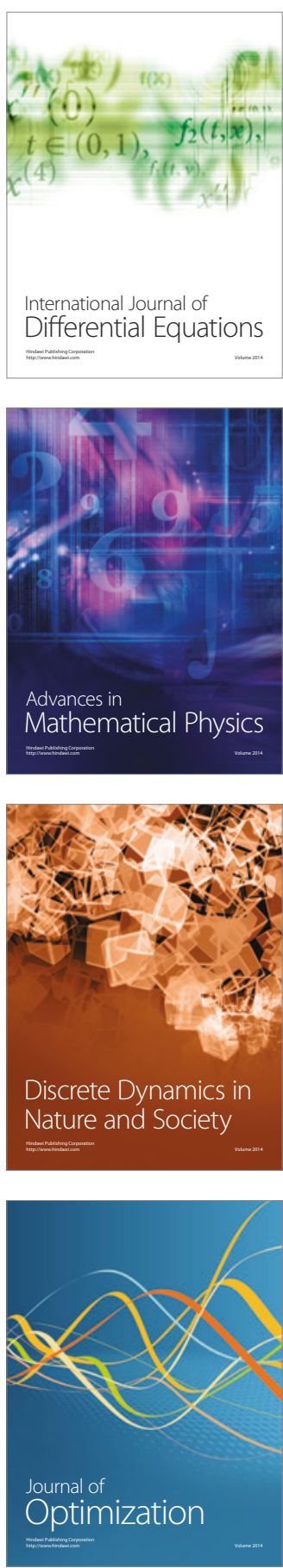